\title{
Influencing medication taking behaviors using automated two-way digital communication: A narrative synthesis systematic review informed by the Behavior Change Wheel
}

\author{
Gemma Donovan ${ }^{1}$ (1), Nicola Hall ${ }^{2}$ (D), Jonathan Ling $^{3}$ (i), \\ Felicity Smith ${ }^{4}$ (1) and Scott Wilkes ${ }^{5}$ \\ 'Faculty of Health Sciences and Wellbeing, School of Pharmacy and Pharmaceutical \\ Sciences, University of Sunderland, UK \\ ${ }^{2}$ Faculty of Medical Sciences, Population Health Sciences Institute, Newcastle \\ University, UK \\ ${ }^{3}$ Faculty of Health Sciences and Wellbeing, University of Sunderland, UK \\ ${ }^{4}$ UCL School of Pharmacy, UK \\ ${ }^{5}$ Faculty of Health Sciences and Wellbeing, School of Medicine, University of \\ Sunderland, UK
}

\begin{abstract}
Purpose. Around half of prescribed medications for long-term conditions are not taken as directed. Automated two-way digital communication, such as text messaging and interactive voice response technology, could deliver interventions to improve medication adherence, and subsequently health. However, exploration of how such interventions may improve medication adherence is limited. This review aimed to explore how automated two-way digital communication can improve medication taking with or without using non-digital intervention components, such as phone calls with healthcare professionals.
\end{abstract}

Methods. A theory-informed narrative synthesis systematic review. Several databases were searched including CINAHL, Embase, Medline, and Web of Science using key words relating to 'medication adherence' and digital communication technologies. The Behavior Change Technique (BCT) coding using the BCT Taxonomy VI and the Behavior Change Wheel were used to identify BCTs delivered within the included interventions.

Results. A total of 3,018 records were screened with 43 study reports included in the review. Four medication-taking behaviors: taking medication, obtaining medication, selftesting, and asking for support were identified as targets for behavior change within the included interventions. Most BCTs within the digital communication component aimed to increase motivation for medication adherence, with non-digital intervention components included to address other medication taking barriers, such as physical and psychological capability.

\footnotetext{
This is an open access article under the terms of the Creative Commons Attribution License, which permits use, distribution and reproduction in any medium, provided the original work is properly cited.

*Correspondence should be addressed to Gemma Donovan, University of Sunderland, Dale I 2 I, Sciences Complex, Wharncliffe Street, Sunderland, Tyne and Wear SRI 3SD, UK (email: gemma.donovan@sunderland.ac.uk)
} 
Conclusion. Automated two-way digital communication can detect barriers to medication adherence by monitoring performance of the taking medication behavior. Monitoring outcomes from taking medication may increase reflective motivation to take medicines. Addressing physical opportunity to taking medication by facilitating the behavior obtaining medication may also increase adherence.

\section{Statement of contribution}

\section{What is already known on this subject?}

- Around half of prescribed medications for long-term conditions are not taken as directed.

- The Capability, Opportunity, and Motivation model of behavior has been used to explain medicationtaking.

- Automated two-way digital communication has been shown to have positive effects on medication adherence.

\section{What does this study add?}

- Identification of four medication taking behaviors targeted using automated two-way digital communication.

- Improved medication adherence was found in studies facilitating the behavior obtaining medication.

- Barriers to taking medication can be detected through behavioral monitoring using two-way digital communication.

\section{Background}

The increasing proportion of people living with long-term conditions (LTCs) and multimorbidity is placing a growing burden on health and social care systems. Data from the UK Health Survey show that $45 \%$ of women and $41 \%$ of men have a longstanding illness (Fat, 2018). Medicines are the most common intervention to treat and manage LTCs; $48 \%$ of adults in the UK take at least one prescribed drug (Moody, Mindell, \& Faulding, 2016). However, we know that $30-50 \%$ of patients with LTCs do not take their medication as directed by their prescriber (World Health Organization, 2003) and that evidence for interventions which tackle nonadherence effectively continues to be elusive. The impact of medication nonadherence is far reaching: a potential reduction in therapeutic effect can lead to patients requiring further intervention with subsequent economic implications (Cutler, Fernandez-Llimos, Frommer, Benrimoj, \& Garcia-Cardenas, 2018). Improving medication adherence can decrease mortality rates in hypertensive patients (Morisky et al., 1983) and reduce hospitalisations in patients with asthma (Trautner, Richter, \& Berger, 1993).

Reviews on the use of a wide range of digital communication technologies for supporting medication adherence have arrived at mixed conclusions. However, many reviews support their potential to enhance medication adherence (Fang, Maeder, \& Bjering, 2016; Lee, Ralston, Beautrais, \& Larkin, 2014; Sarabi, Sadoughi, Orak, \& Bahaadinbeigy, 2016; Sarkar \& Sivashankar, 2015; Vervloet et al., 2012) and that they are acceptable to patients (Anglada-Martinez et al., 2015; Park, Howie-Esquivel, \& Dracup, 2014). A meta-analysis has also found that text messages can improve adherence to medication (Thakkar, Kurup, \& Laba, 2016). Some reviewers have also concluded that two-way communication may be more effective than one-way. Software can also be used to send messages automatically at appropriate times and respond to patients using pre-set algorithms, providing the opportunity for cheap and low-burden interventions (Iribarren, Brown, \& Giguere, 2017). This review was intended for use as a basis to design a new intervention using such a system, Simple Telehealth (Simple Shared Healthcare Ltd, 
2020). This uses Short Message Service (SMS) technolgy and was already available for use in the National Health Service (NHS). As such technology is relatively new, we expanded our review to examine evidence that could be adapted from older technologies, such as Interactive Voice Response (IVR) systems and pagers. However, we currently lack explanations as to bow automated two-way digital communication interventions might work to improve medication adherence.

Reasons for medication non-adherence are also complex. Literature in this area describes a range of theories and models to explain and predict medicating taking behaviors (Easthall \& Barnett, 2017). Behavioral frameworks, such as the Capability, Opportunity, and Motivation for Behavior (COM-B) model (Michie, van Stralen, \& West, 2011), can provide a comprehensive lens through which to examine the problem of medication nonadherence (Easthall \& Barnett, 2017; Jackson, Eliasson, Barber, \& Weinman, 2014). The COM-B model describes behaviors as being influenced by an individual's physical and psychological capability to perform a behavior, their physical and social opportunity to engage in the behavior, and their reflective and automatic motivation for conducting the behavior (Michie et al., 2011). The Behaviour Change Wheel (BCW) uses the COM-B model as a framework for characterizing and designing behavior change interventions, including a mapping process to 'intervention functions' that then guide the selection of Behaviour Change Techniques (BCTs) (Michie, van Stralen, \& West, 2011). A previous review has mapped medication taking to the COM-B model (Jackson et al., 2014), and a more recent review mapped BCTs used in automated two-way interventions for cardio-metabolic conditions using meta-regression (Kassavou \& Sutton, 2018). Currently, a review which considers a full range of LTCs is lacking.

Text messaging alone is also unable to address more practical barriers to medication adherence, such as difficulties with patients accessing medicines from packaging. The use of digital communication may be optimised when used in addition to other medicationrelated support (Ciciriello, Johnston, \& Osborne, 2013; Fenerty, West, Davis, Kaplan, \& Feldman, 2012; Granger \& Bosworth, 2011; Hamine, Gerth-Guyette, Faulx, Green, \& Ginsburg, 2015; Mistry, Keepanasseril, \& Wilczynski, 2015; Park, Howie-Esquivel, \& Dracup, 2014), such as communication with healthcare professionals in follow-up telephone calls or face-to-face consultations. There are no reviews which consider these non-digital intervention components, so it is currently unknown as to how these two elements may interact.

\section{Objectives}

The aim of this review was to explore how two-way automated digital communication interventions, with or without non-digital components, can improve medication adherence and clinical outcomes for patient participants with long-term conditions. This was achieved by 1) coding the BCTs, their target behavior, and delivery mode relating to medication adherence using intervention descriptions, 2) mapping the BCTs and behaviors to the most likely COM-B component influenced for medication taking, and 3) comparing studied outcomes with the behavioral mechanism and delivery mode to identify how interventions may work to increase medication adherence and/or clinical outcomes for patients. 


\section{Methods}

We used a narrative synthesis systematic review method to evaluate the potential mechanisms of effectiveness for automated two-way digital communication interventions on medication adherence and clinical outcomes using BCT coding and the BCW. A narrative synthesis review method was chosen as we anticipated high heterogeneity based on previous reviews (Kassavou \& Sutton, 2018; Thakkar et al., 2016) and our broad inclusion criteria. This article focuses on one research question from a larger systematic review protocol registered on the PROSPERO Database prior to completion (CRD42017069290) and relates specifically to the use of the COM-B model to characterise and explain the effects of automated two-way patient contact interventions on medication adherence. The PRISMA statement 2020 (Page, McKenzie, \& Bossuyt, 2020) was used to prepare this report, and the full checklist is available in the Supplementary Materials.

\section{Eligibility criteria}

Studies were eligible for inclusion if their participants were adults (over 18 years) who were self-managing their medication in their own home with any LTC, and the aim of the intervention was to affect medication adherence. Only interventions delivered in highincome countries as classified by the World Bank (The World Bank, 2020) were considered to maximise transferability of findings into a new intervention in the UK NHS. The main intervention needed to include automated two-way digital communication in its delivery. The digital communication technologies of interest were IVR or text messaging using SMS or pagers. Interventions could also have non-digital intervention components in addition to the use of the automated two-way digital communication. Any study comparator was considered. Studies were included if they reported outcomes relating to either medication adherence and/or clinical outcomes relevant to the LTC under study. Examples included clinical measurements such as blood pressure or symptom assessment tools. Only pilot or feasibility studies were excluded as these would not be powered to provide a robust evaluation of the outcome measures. Only studies published in English were examined. A full list of eligibility criteria can be found in Appendix S1.

\section{Information sources}

Databases searched included Medline, CINAHL, PsycARTICLES, Psychology and Behavioral Sciences collection and International Pharmaceutical Abstracts using EBSCO Host, and Embase using Ovid. Web of Science, PubMed, and Cochrane Library were searched separately. Gray literature was also searched including the Simple Telehealth Network, British Library EthOS, and Trove and Opengrey.eu. The initial search was conducted in May-June 2017 with no lower date limit and was updated in September 2020. Reference lists from similar reviews were also evaluated for inclusion (AngladaMartinez et al., 2015; Ciciriello et al., 2013; Dekoekkoek, Given, \& Given, 2015; Fang et al., 2016; Fenerty et al., 2012; Fjeldsoe, Marshall, \& Miller, 2009; Granger \& Bosworth, 2011; Hamine, Gerth-Guyette, Faulx, Green, \& Ginsburg, 2015; Lee et al., 2014; Linn, Vervloet, van Dijk, Smit, \& Van Weert, 2011; Mistry et al., 2015; Nieuwlaat, Wilcyzynski, \& Navarro, 2014; Park et al., 2014; Sarabi et al., 2016; Sarkar \& Sivashankar, 2015; Tao, Xie, Wang, \& Wang, 2015; Thakkar et al., 2016; Vervloet et al., 2012; Wald, Butt, Bestwick, \& Bestwick, 2015). Supplementary documents to support BCT coding were obtained where possible, including published development studies and by contacting authors directly. 


\section{Search}

Thesaurus terms were used where available, supplemented by a range of key terms to search titles and abstracts. Search terms were developed to combine literature examining medication adherence and digital communication technologies. The selection and combination of terms was partially informed by similar reviews (Anglada-Martinez et al., 2015; Ciciriello et al., 2013; Dekoekkoek et al., 2015; Fang et al., 2016; Fenerty et al., 2012; Fjeldsoe et al., 2009; Hamine et al., 2015; Lee et al., 2014; Linn et al., 2011; Mistry et al., 2015; Nieuwlaat et al., 2014; Park et al., 2014; Sarabi et al., 2016; Sarkar \& Sivashankar, 2015; Tao et al., 2015; Thakkar et al., 2016; Vervloet et al., 2012; Wald et al., 2015). A full search strategy is available in Appendix S1.

\section{Study selection}

Two reviewers (GD and $\mathrm{NH}$ ) were involved in the initial study selection process. Titles and abstracts of the result list were screened independently by GD and NH to compile a list of articles for full text review. Discrepancies between the reviewers were discussed and agreed. Full text articles were then obtained where possible and again screened independently by GD and NH to create a list of studies for inclusion within the review. The updated search was conducted by GD based on discussions from the original study selection processes.

\section{Data collection processes}

Data were extracted from each study on design characteristics, participant characteristics, intervention characteristics, intervention delivery details, and study outcomes. A data extraction tool was created using Google Forms (Google LLC, 2018) (see Appendix S2) and exported as an Excel spreadsheet to facilitate the comparison of inputs between both reviewers and the tabulation of results. The form was piloted by GD and then revised prior to extraction. Data entry was completed by GD and $\mathrm{NH}$ independently, with discrepancies resolved through discussion. Each study was assessed for quality using the Mixed-Methods Appraisal Tool (MMAT) v1 (Pluye, Robert, Cargo, \& Proposal, 2011). Data extraction for studies identified in the updated search was completed by GD, informed by the original discussions.

\section{Data analysis}

Studies were first analyzed by describing study characteristics and outcomes. BCT coding was conducted for each individual study on multiple levels. Text describing the intervention was highlighted and coded for the inclusion of BCTs using the BCT Taxonomy v1 (Michie, Richardson, \& Johnston, 2013), the behavior which the BCT targeted and the BCT mode of delivery (automated two-way digital communication component or non-digital component) in NVivo 12 (QSR International Pty Ltd. NVivo, 2018). This created 'layers' of coding which could be combined using the 'overlapping' search query function.

Coding was conducted independently by GD and NH, with differences resolved through discussion. A coding manual is available as Appendix S3. To support the coding of behaviors, potential medication self-management behaviors were determined a priori by the study team based on experience as healthcare professionals and following guidance in the BCW (Michie, Atkins, \& West, 2014). Using guidance in the BCW, the most likely 
intervention functions and COM-B components that interventions seemed to use to influence medication taking were identified by the review authors, informed by study author explainations within journal articles where available. The identified BCW mechanisms were then charted against medication adherence and clinical outcomes to evaluate whether these mechanisms seemed to influence medication adherence and clinical improvements. This was done by considering the presence or absence of $\mathrm{BCW}$ mechanisms and whether outcomes from these interventions were improved, not improved, or had unclear results by vote counting the relevant included studies. Outcome categories were decided based on a combination of outcomes as reported in the original articles and consideration of the validity of outcomes in the context of study quality appraisal, in particular the method of medication adherence measurement. This is discussed as part of the results. The frequency with which BCTs were directed against the different behaviors was determined using searches of overlapping coding facilitated by NVivo 12 (QSR International Pty Ltd. NVivo, 2018). Analysis was completed separately for the automated digital communication and non-digital intervention components to consider how these two delivery formats may be contributing to overall intervention outcomes.

\section{Results}

\section{Study selection}

A summary of the screening and filtering of search results for the original search is provided in the PRISMA diagram (Figure 1). No additional records were found using the gray literature, but a further 25 were found from the pearling of references from other published reviews. Forty-one papers covering 34 studies were included from the original search. A further two studies and associated articles were identified from the updated search (Johnston, van der Kop, \& Smillie, 2018; Kuusalo, Sokka-Isler, \& Kautiainen, 2020). Most of the studies examined the impact of interventions on medication adherence $(n=35)$ and 20 examined clinical outcomes.

\section{Study characteristics}

A summary of the study characteristics can be found in Table 1. Most included studies were randomised controlled trials $(n=28)$. Studies were predominantly conducted in the United States $(\mathrm{n}=30)$. The number of participants included within studies ranged from 40 to 21,752. A summary of the quality appraisal using the MMAT can be found in Appendix S4. Most of the RCTs were of good quality, usually only missing the 'lack of allocation concealment' criteria. Patient concealment is not possible with this type of intervention; however, some described concealment of investigators.

\section{Intervention characteristics}

A summary of the intervention characteristics and detailed results can be found in the spreadsheet Appendix S5. The automated two-way digital communication technologies examined included IVR $(n=19)$, SMS $(n=11)$, and pager devices $(n=2)$. Four studies used a combination of technologies, either to complement each other or to offer patient participants a choice on which mode of delivery they would prefer. Fifteen studies 
Identification for studies via databases

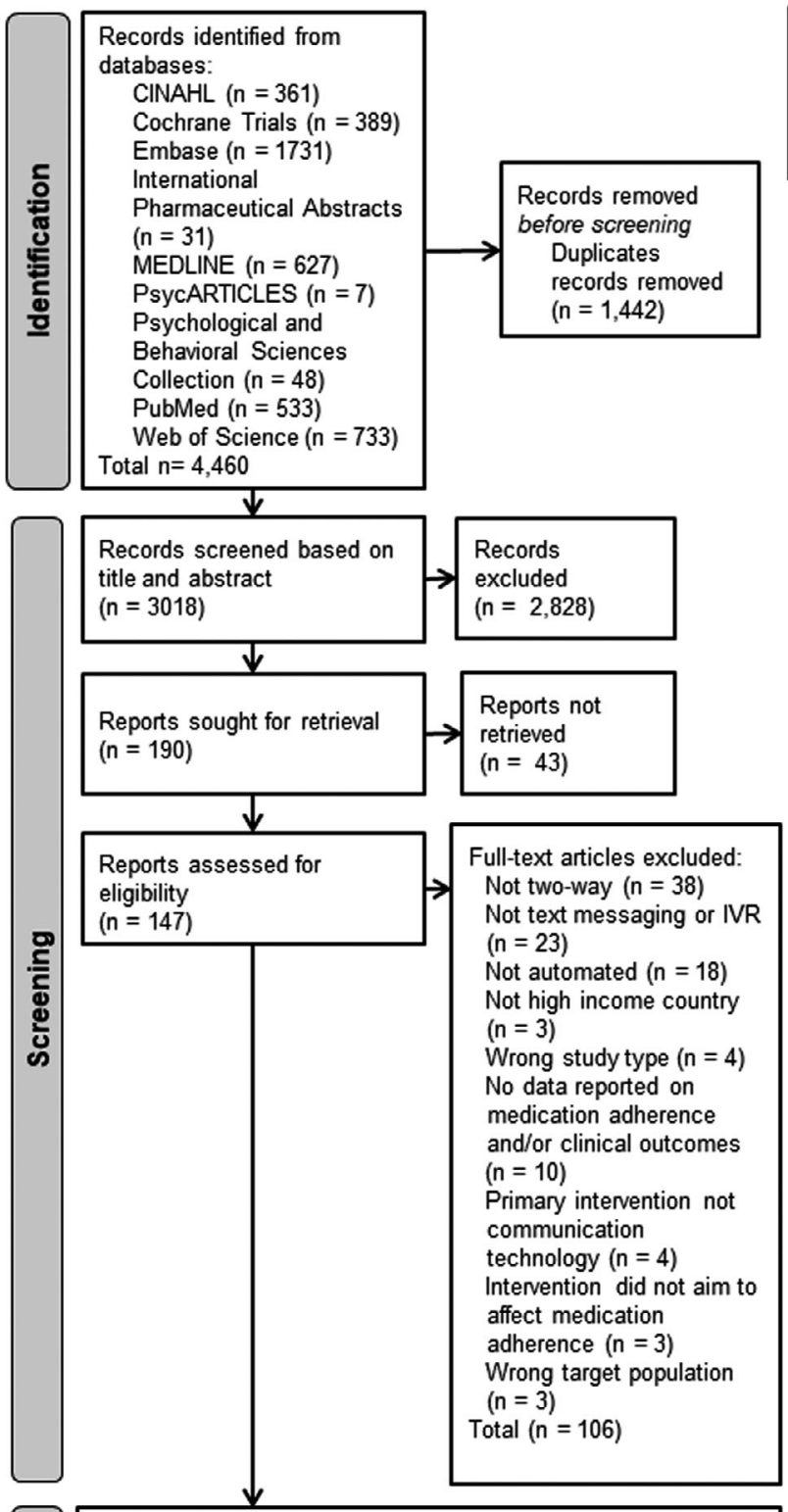

Identification for studies via other methods

Records identified from:

Citation searching

( $n=25)$

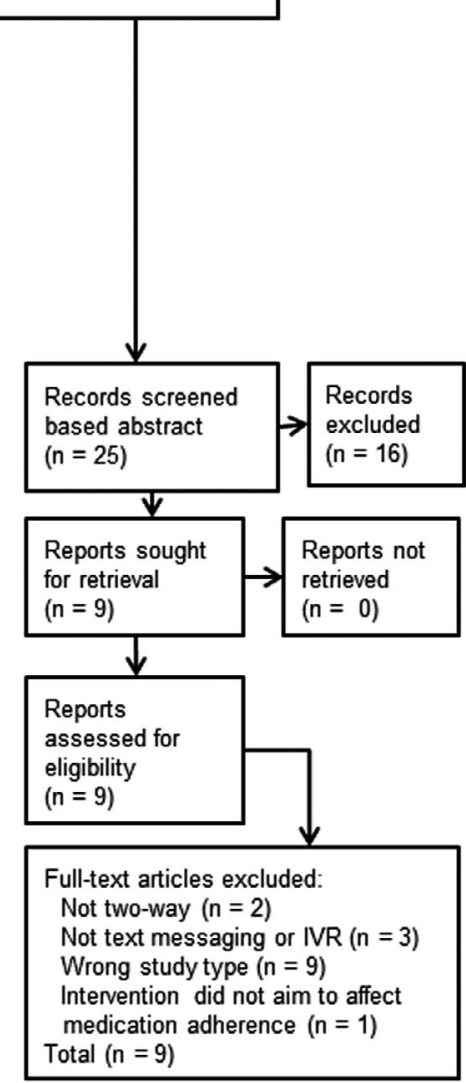

New studies identified for inclusion from updated search $(n=2)$

Total studies included in review $(n=34)$

Reports of total included studies $(n=41)$ (June 2017)

Total studies included in review $(n=36)$

Reports of total included studies $(n=43)$ (September 2020)

Figure I. PRISMA Diagram representing selection of included studies. 


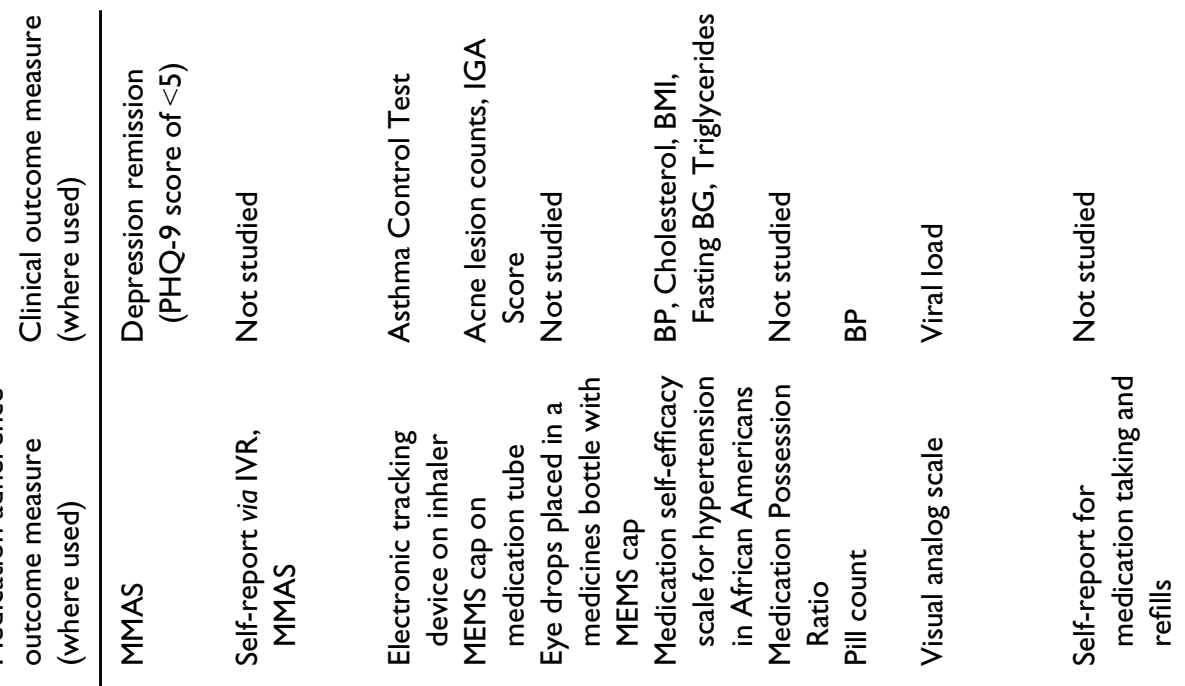

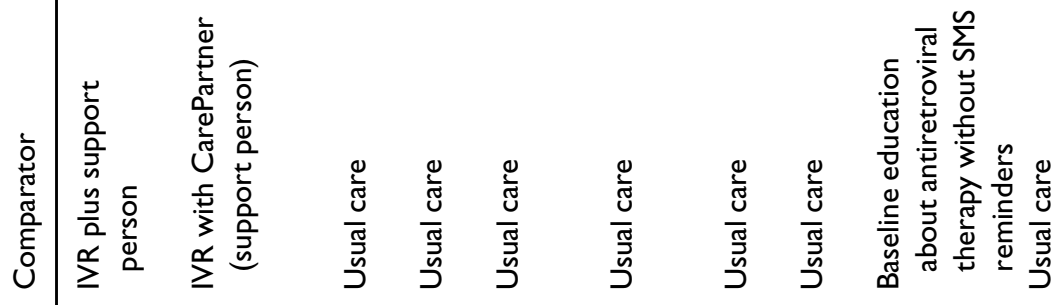

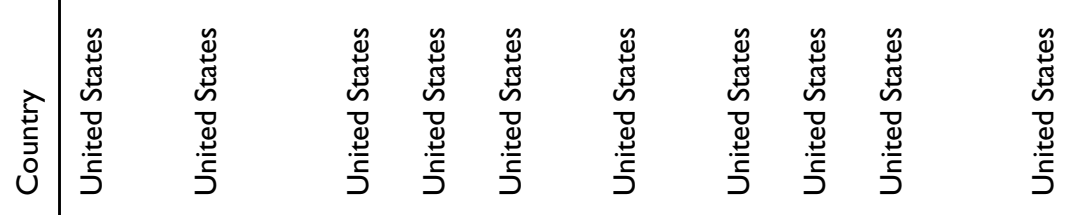

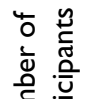

ह 宽

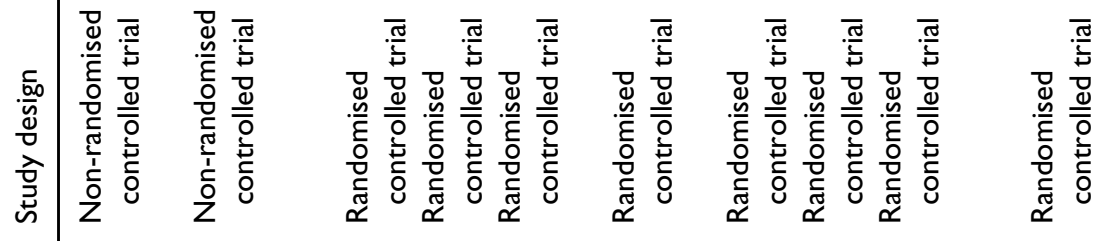

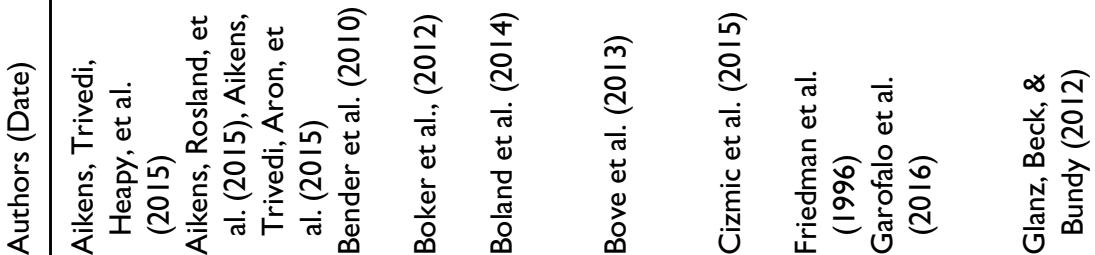


Digital communication for medication taking 9

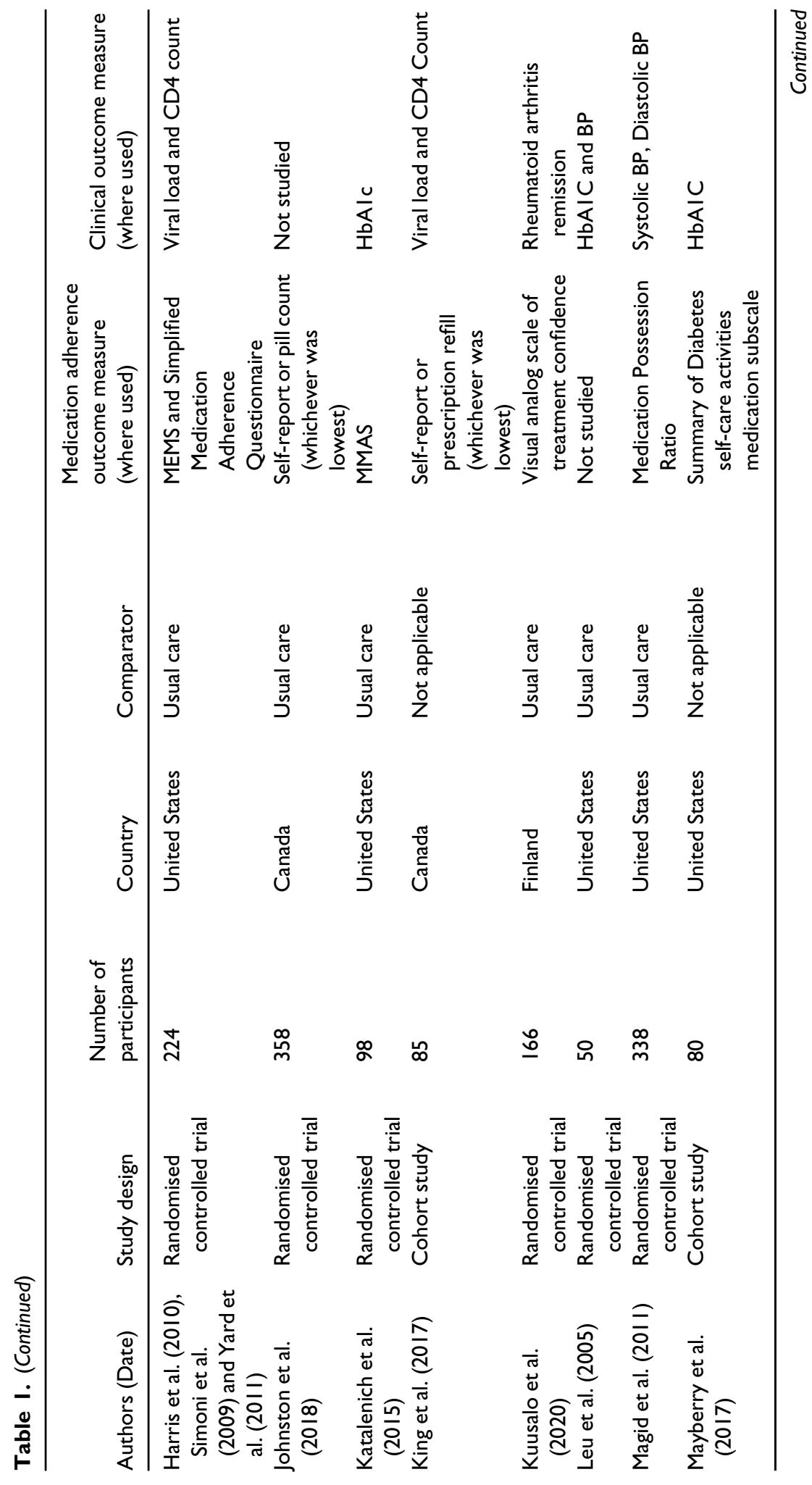



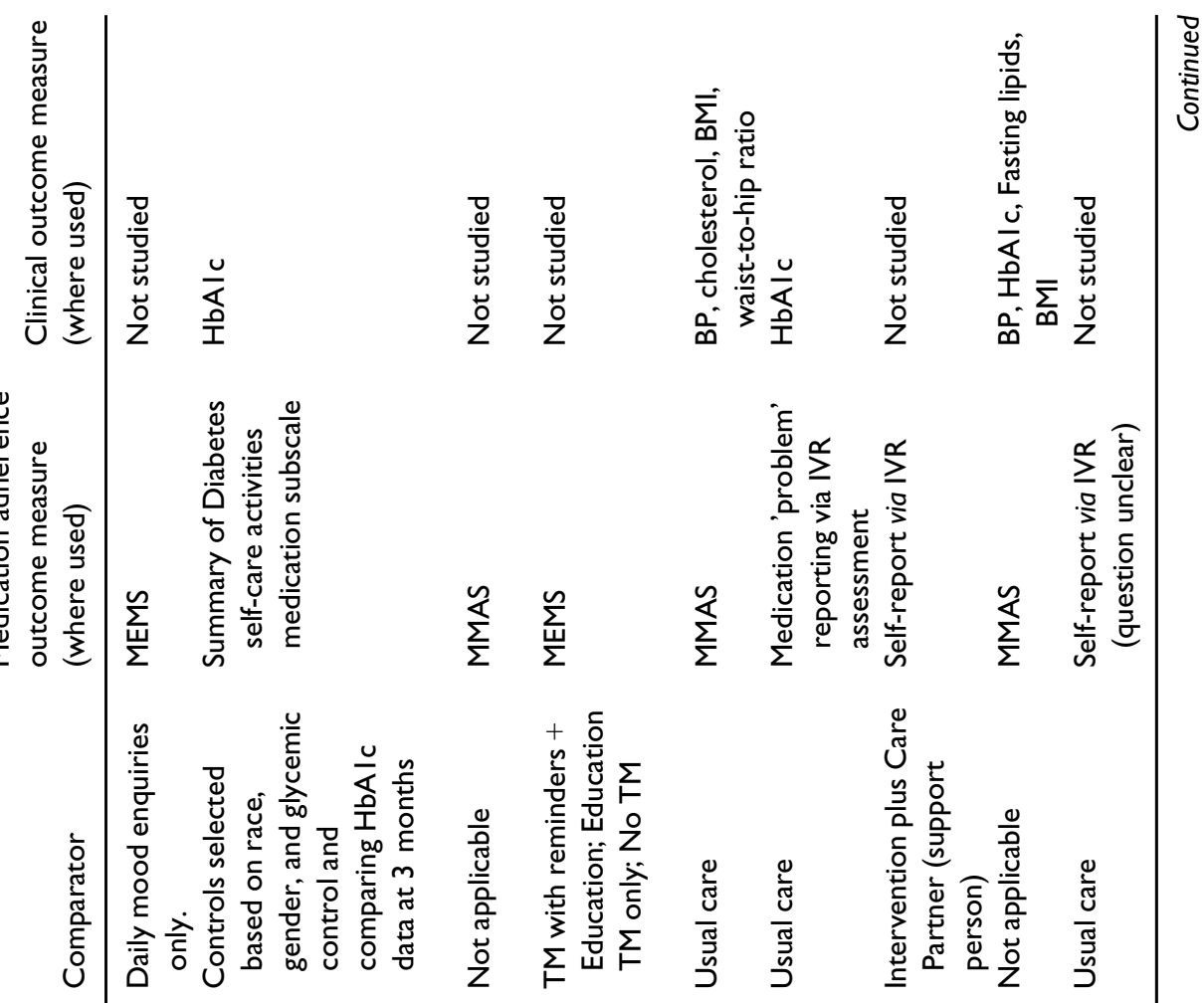

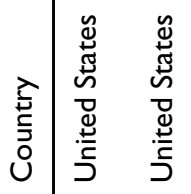

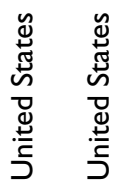

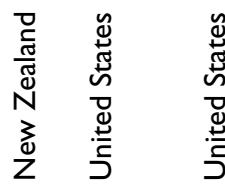

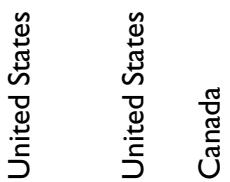

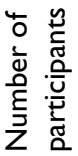

i̊ $\infty$

ホ

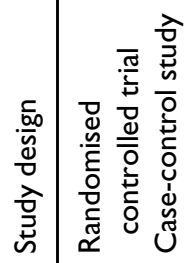

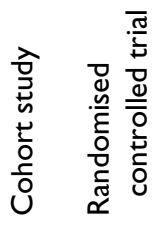

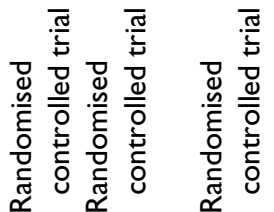

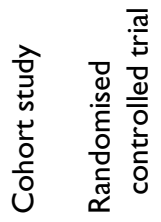

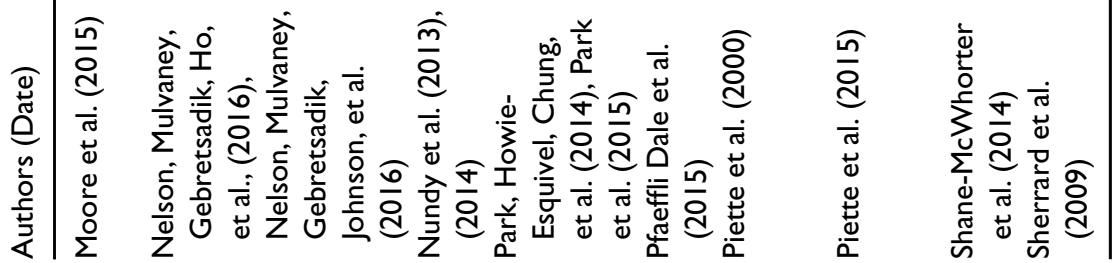




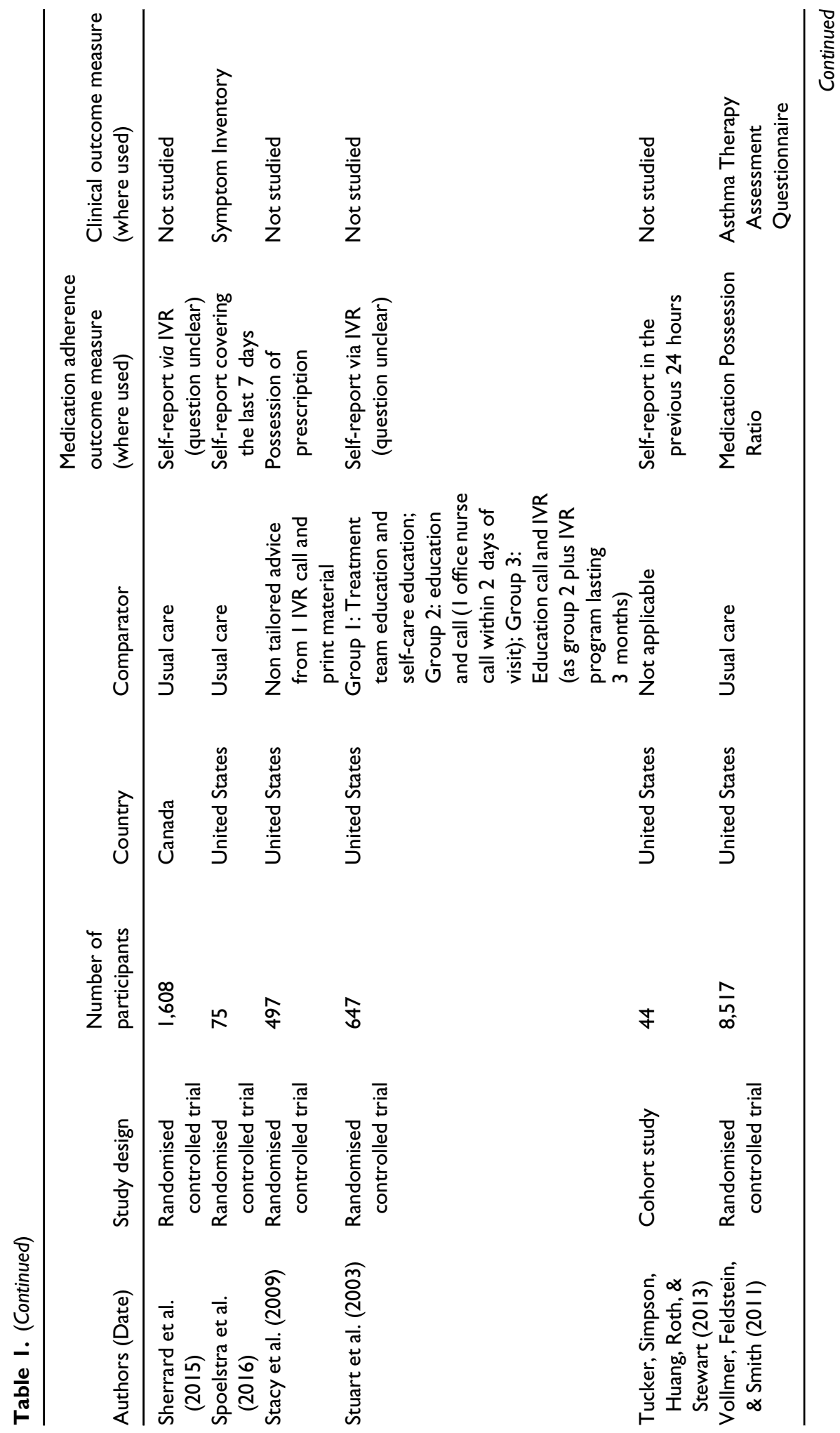




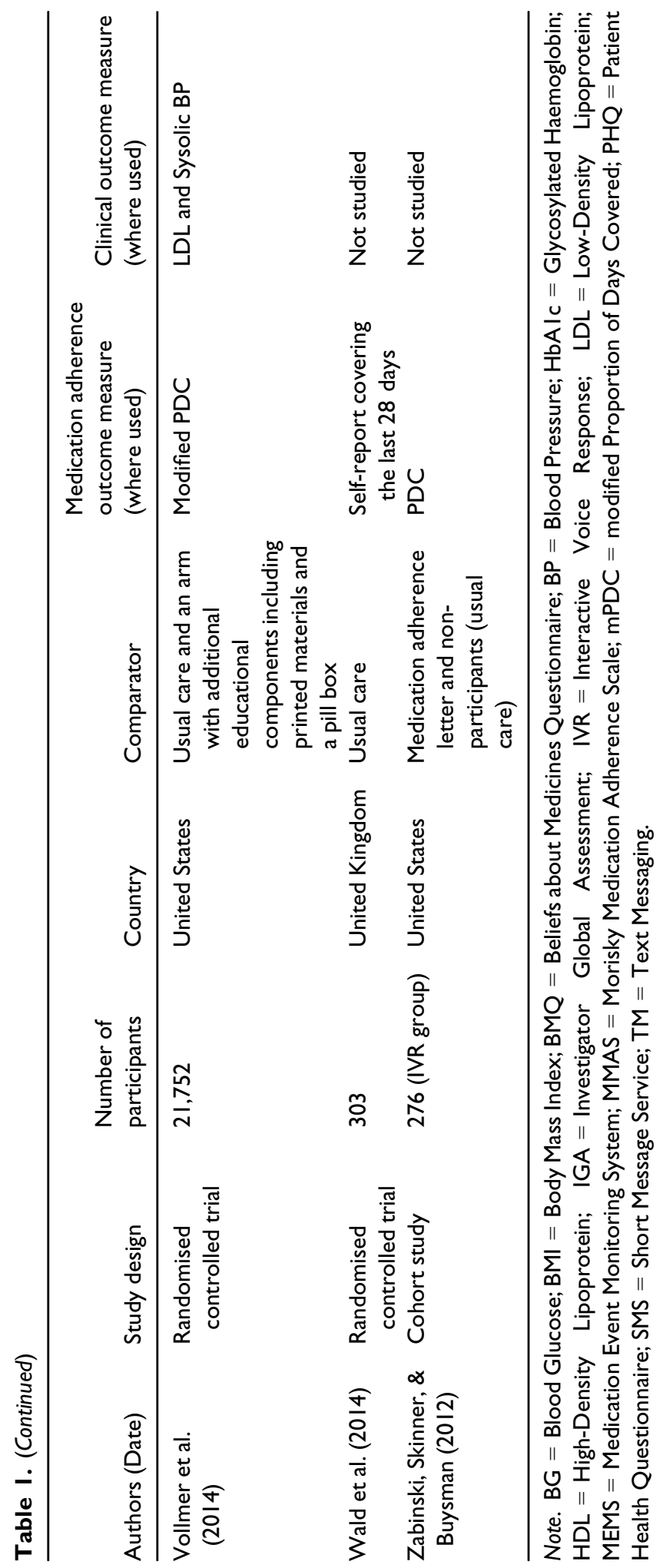


included at least one non-digital intervention component alongside the use of automated two-way digital communication.

Length of intervention ranged from a one-off interactive call to 12 months of two-way communication. The most common intervention aims were to improve adherence to an existing therapy $(n=23)$, followed by promoting adherence to a new therapy $(n=11)$. A small number of studies aimed to detect non-adherence to medicines $(n=2)$ or maintain adherence to medication $(n=1)$. In one case, the aim of the intervention in relation to affecting medication adherence was unclear.

Most studies delivered interventions for a single LTC. The most common of these was cardiovascular disease $(n=13)$. Other LTCs included diabetes $(n=8)$, HIV/ AIDS $(n=6)$, depressive disorders $(n=4)$, osteoporosis $(n=1)$, cancer $(n=2)$, asthma $(n=2)$, chronic obstructive pulmonary disease $(n=1)$, tuberculosis $(n=1)$, glaucoma $(n=2)$, rheumatoid arthritis $(n=1)$, and acne $(n=1)$.

\section{Study results}

Of the 36 studies included in the review, most $(n=34)$ provided outcomes for medication adherence and of these, 19 found improvement. Seven studies had unclear findings on medication adherence, and eight found no improvement. Two studies only assessed clinical outcomes, and an additional 18 provided clinical outcome data alongside medication adherence. Clinical outcomes were improved in seven studies but mostlu either did not find an improvement $(n=9)$ or had unclear outcomes $(n=4)$, even if there were improvements in medication adherence. Only four studies reported clearly positive outcomes in medication adherence and clinical outcomes.

\section{A medication behavioral map}

A summary of the behaviors targeted, the BCTs which were used, the delivery format of the BCTs, and outcomes for the included studies is available in the spreadsheet Appendix S5. Four medication-related behaviors were targeted for change within interventions: taking medication ( $\mathrm{n}=35$ studies); obtaining medication $(\mathrm{n}=10)$; self-testing $(\mathrm{n}=9)$; and asking for support $(\mathrm{n}=7)$. We describe the relationships between these behaviors in Figure 2 with a behavioral map. While there was often little detail about the support patients asked for, or what was delivered as the interventions included in the review all aimed to affect medication adherence, we have inferred that the behaviur asking for support would be linked to this outcome. Seventeen studies targeted just one behavior, 13 targeted two behaviors, and six targeted three behaviors.

The number of BCTs included within interventions ranged from 1 to 10; however, there did not seem to be a relationship between the number of BCTs included and study outcomes. The average number of BCTs included in interventions with improvement in medication adherence was 4.8 versus 4.6 in those without improvement, and this was 5.6 BCTs in both groups for clinical outcomes. The most frequently included BCTs were Prompts/Cues $(\mathrm{n}=19)$ and Monitoring of bebavior by otbers witbout feedback $(\mathrm{n}=19)$, which were mostly included within the automated digital communication component. Figure 3 summarises the number of study interventions targeting each medication-taking behavior and the BCTs used for the automated digital communication component of interventions. Social support (unspecified) $(\mathrm{n}=17)$ and Biofeedback $(\mathrm{n}=12)$ were the most common BCTs included as part the non-digital intervention components (see Figure 4). 


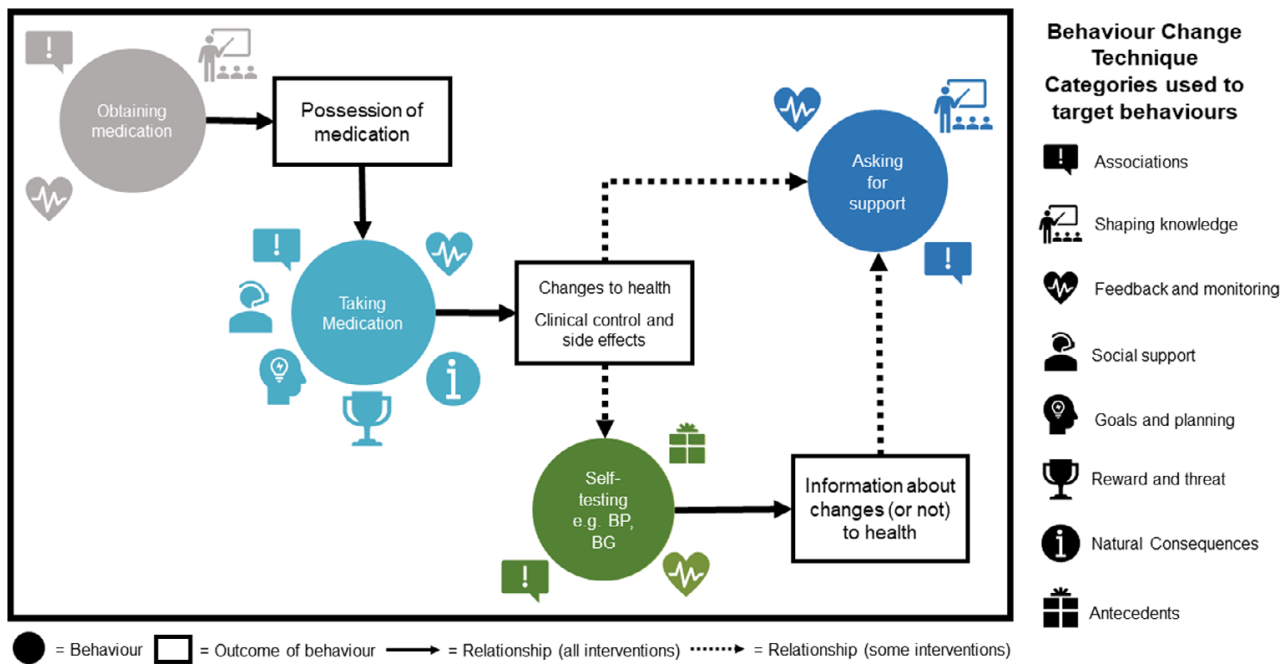

Figure 2. A map of the behaviors targeted within interventions and the Behavior Change Technique (BCT) categories used to target these within included interventions.

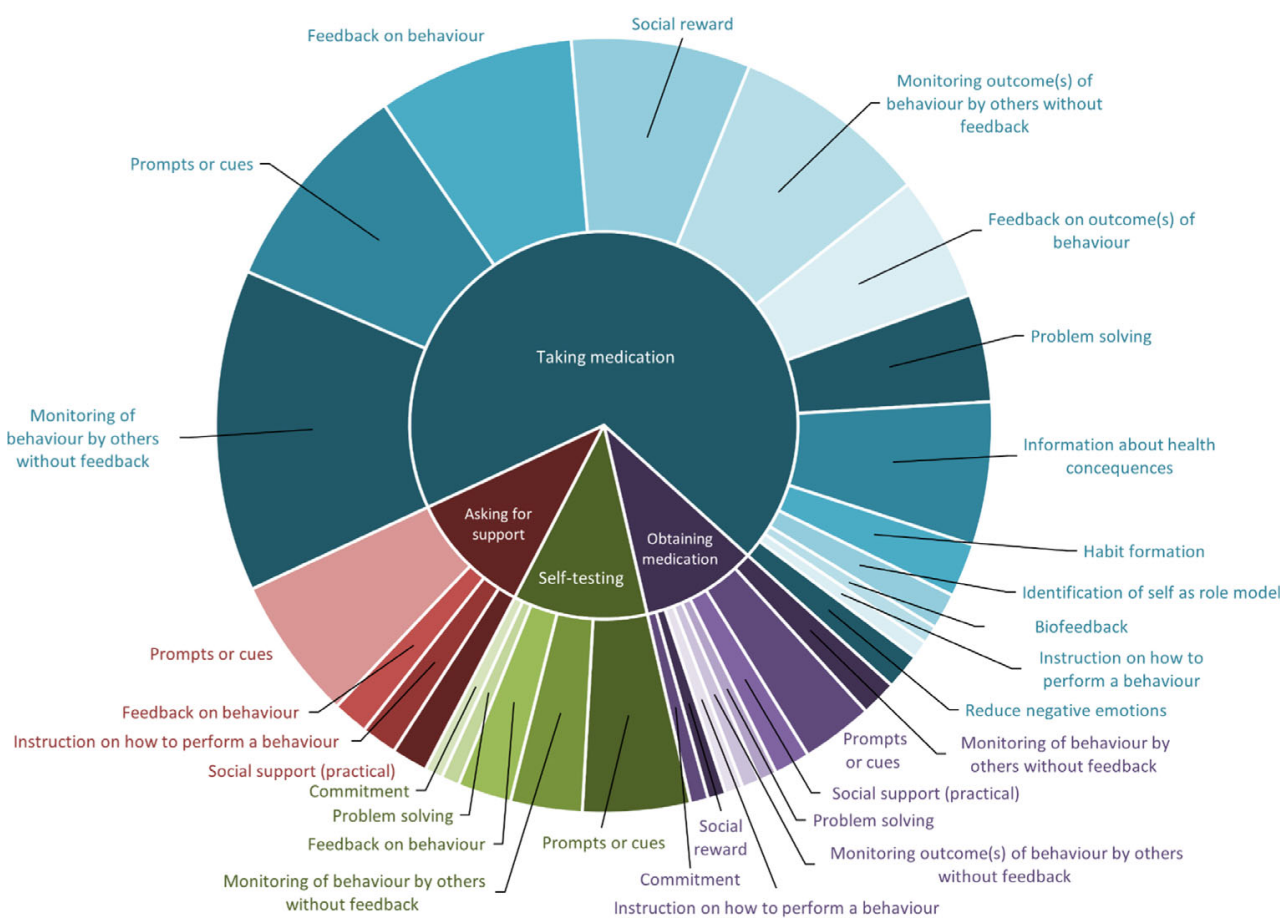

Figure 3. Sunburst diagram representing the frequency of Behavior Change Technique delivery against medication taking behaviors for automated two-way digital communication intervention components.

There were also examples of integration between the automated two-way digital communication and non-digital components. This included interventions delivering different BCTs using different components or delivering the same BCT using both 


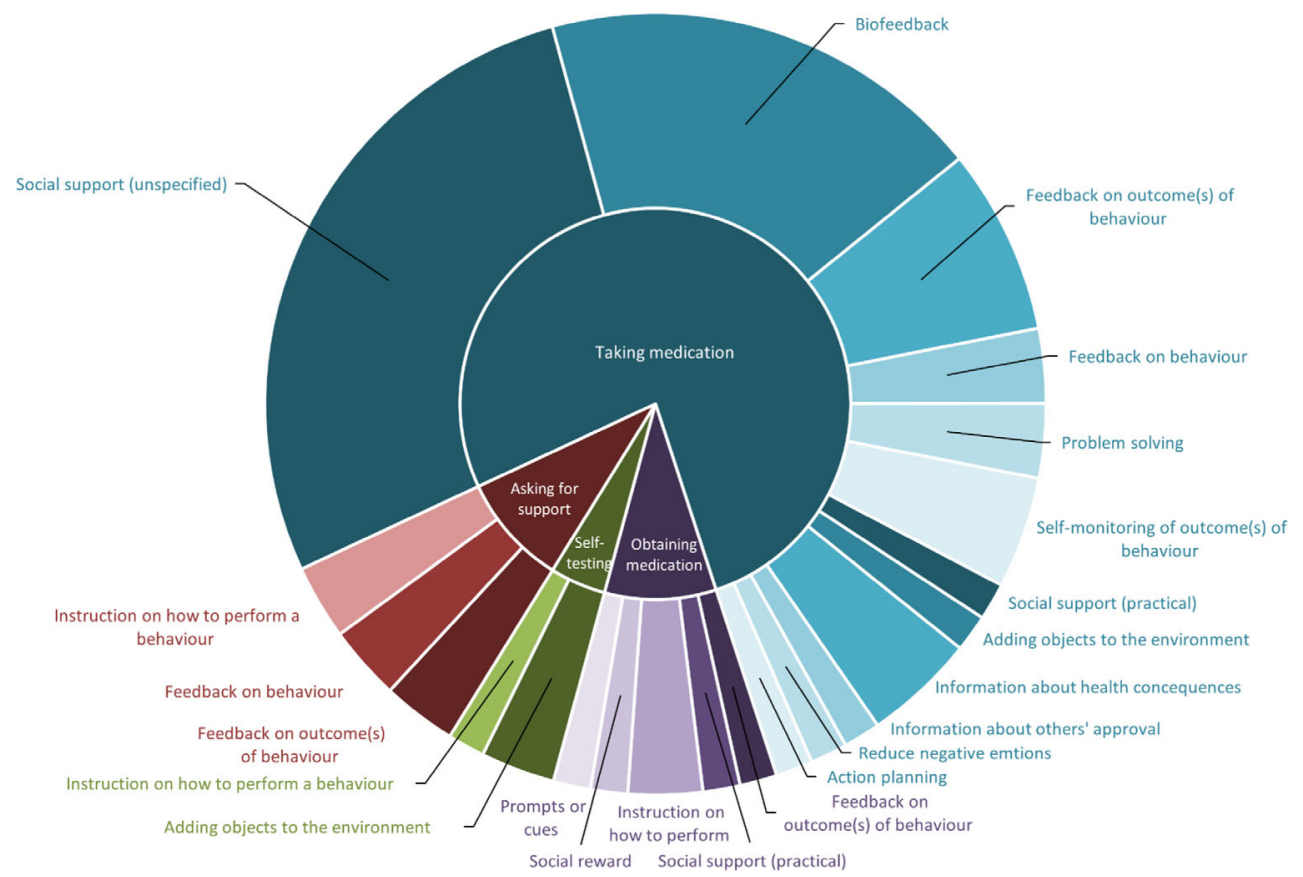

Figure 4. Sunburst diagram representing the frequency of Behavior Change Technique delivery against medication taking behaviors for non-digital communication intervention components.

components. This has been highlighted in the color coding in Appendix S5. The following results are organised into how the behavioral components of interventions seemed to influence capability, opportunity, and motivation for the different medication taking behaviors identified.

Obtaining medication to remove the physical opportunity barrier to taking medication Within our behavioral map (see Figure 2), performing the behavior obtaining medication is a pre-requisite to the behavior taking medication. Without physical opportunity, taking medication is unlikely to occur. This behavior was targeted by both digital communication and non-digital components (see Figure 3 and Figure 4). BCTs were identified as targeting obtaining medication where interventions influenced activities such as ordering medication from a prescriber and/or collecting it from a pharmacy. The most common BCT used to target this behavior was Instruction on bow to perform a behavior using automated digital communication. Interventions that included BCTs to target obtaining medication had an almost universally positive impact on medication adherence with $90 \%$ of studies targeting this behavior finding improvements. This suggests that this behavior is a good target where physical opportunity is a barrier to taking medication.

Increasing automatic motivation to improve taking medication

Interventions which targeted taking medication in our behavioral map (see Figure 2) are those targeting self-administering a medication. Interventions which targeted this behavior seemed to aim to improve medication adherence by supporting the formation 
of habit via automatic motivation. Although the Habit formation BCT has a narrow definition in the BCT Taxonomy v1, the repetition required to form a habit could be encouraged using a range of BCTs. For example, the BCT Prompts/Cues was frequently used to target taking medication with one-way automated messages. An example message would be, "It's time to take your medication". Eleven studies include the use of one-way Prompts/Cues (Boker, Feetham, Armstrong, Purcell, \& Jacobe, 2012; Boland et al., 2014; Garofalo et al., 2016; Harris, Lehavot, \& Huh, 2010; Katalenich, Shi, \& Liu, 2015; Leu, Norris, Hummel, Isaac, \& Brogan, 2005; Moore, Poquette, \& Casaletto, 2015; Nundy, Dick, Solomon, \& Peek, 2013; Nundy et al., 2014; Park, Howie-Esquivel, Chung, \& Dracup, 2014; Park, Howie-Esquivel, Whooley, \& Dracup, 2015; Simoni, Huh, \& Frick, 2009; Spoelstra, Given, \& Sikorskii, 2016; Wald, Bestwick, Raiman, Brendell, \& Wald, 2014; Yard, Huh, King, \& Simoni, 2011). However, the inclusion of this BCT did not have a consistent effect on medication adherence outcomes with only five of these reporting improvements in medication adherence.

Increasing awareness of the medication taking behavior through medication monitoring with or without feedback may have also been used to increase the medication taking habit. Monitoring behavior and providing feedback was automated in some studies (Aikens, Rosland, \& Piette, 2015; Aikens, Trivedi, Aron, \& Piette, 2015; Garofalo et al., 2016; Mayberry, Mulvaney, \& Johnson, 2017; Moore et al., 2015; Piette, Weinberger, McPhee, Mah, \& Kraemer, 2000; Sherrard, Duchesne, Wells, Kearns, \& Struthers, 2015; Stuart, Laraia, Ornstein, \& Nietert, 2003) while others used non-digital components, such as follow-up calls, to provide feedback (Leu et al., 2005; Sherrard, Struthers, Kearns, Wells, \& Mesana, 2009), and two studies used a combination (Aikens, Trivedi, Heapy, Pfeiffer, \& Piette, 2015; Nundy et al., 2013, 2014). There was evidence that providing feedback on behavior seemed to be slightly more linked to results for improved medication adherence, with $43 \%$ of studies providing feedback finding improvements in medication adherence compared to $38 \%$ of studies which monitored medication taking without feedback. Some studies also included the BCT Social reward with 60\% of studies including this BCT (Aikens, Trivedi, Heapy, et al., 2015; Garofalo et al., 2016; Mayberry et al., 2017; Nundy et al., 2013, 2014; Piette et al., 2000; Stacy, Schwartz, Ershoff, \& Shreve, 2009) reported an improvement in medication adherence.

Five studies used a combination of both the feedback and no feedback BCTs, usually with feedback only provided where medication nonadherence was identified. Four of these studies found improvements in medication adherence (Aikens, Rosland, et al., 2015; Aikens, Trivedi, Aron, et al., 2015; Nundy et al., 2013, 2014; Sherrard et al., 2009, 2015). This ability to detect nonadherence and follow-up with patients may, therefore, be beneficial. A small number of studies $(\mathrm{n}=8)$ also delivered the BCT Problem Solving (Aikens, Rosland, et al., 2015; Aikens, Trivedi, Aron, et al., 2015; Mayberry et al., 2017; Nelson, Mulvaney, Gebretsadik, Ho, et al., 2016; Nelson, Mulvaney, Gebretsadik, Johnson, \& Osborn, 2016). All except the study by Aikens, Trivedi, Heapy, et al. (2015) reported improvements in medication adherence.

\section{Increasing reflective motivation to improve medication taking}

Interventions often seemed to aim to increase reflective motivation by attempting to persuade patient participants that medication taking is a 'good' thing to do. This was attempted in eight studies using one-way communication delivering the BCT Information about bealth consequences directed at the behavior taking medication (Bender, Apter, \& Bogen, 2010; Cizmic, Heilmann, Milchak, Riggs, \& Billups, 2015; Friedman, Kazis, \& Jette, 
1996; Mayberry et al., 2017; Moore et al., 2015; Nelson, Mulvaney, Gebretsadik, Ho, et al., 2016; Nelson, Mulvaney, Gebretsadik, Johnson, et al., 2016; Pfaeffli Dale et al., 2015; Spoelstra et al., 2016). In all cases, this was delivered not only using the digital communication component but also supplemented via non-digital intervention components, such as face-to-face consultations (Cizmic et al., 2015; Pfaeffli Dale et al., 2015; Spoelstra et al., 2016). As medicines can have both positive (health improvement) and negative (side effects) consequences, we have differentiated these in the online Supplementary Materials. Including this BCT did not, however, seem to have an effect on study outcomes with only $50 \%$ of studies reporting an improvement in medication adherence (Bender et al., 2010; Cizmic et al., 2015; Friedman et al., 1996; Mayberry et al., 2017).

Asking patients to actively monitor the health effects of taking medication could be used to influence their reflective motivation for medicine taking. For some LTCs, this monitoring requires the introduction of another behavior, which we have called selftesting (see Figure 2). Examples included in this review were blood pressure (BP) or blood glucose (BG) home testing. Completing this self-testing was coded as the Biofeedback BCT and was incorporated into 12 of our included studies (Aikens, Rosland, et al., 2015; Aikens, Trivedi, Aron, et al., 2015; Bove et al., 2013; Katalenich et al., 2015; Leu et al., 2005; Magid, Ho, \& Olson, 2011; Nelson, Mulvaney, Gebretsadik, Ho, et al., 2016; Nelson, Mulvaney, Gebretsadik, Johnson, et al., 2016; Nundy et al., 2013, 2014; Piette, Striplin, \& Marinec, 2015; Piette et al., 2000; Shane-McWhorter, Lenert, \& Petersen, 2014; Vollmer, Owen-Smith, \& Tom, 2014). Where self-testing required the use of a device (e.g. blood glucose monitor), then this was classed as a non-digital intervention component; however, three studies used automated two-way digital communication to directly assess outcomes of taking medication using symptom assessment for depression (Aikens, Trivedi, Heapy, et al., 2015), asthma (Bender et al., 2010), and rheumatoid arthritis (Kuusalo et al., 2020).

Some interventions combined the use of Biofeedback with the BCT Self-monitoring of the outcomes of behavior (Aikens, Rosland, et al., 2015; Aikens, Trivedi, Aron, et al., 2015; Nundy et al., 2013, 2014; Piette et al., 2000). This was delivered where patients were asked to keep records of home monitoring and only submit summaries of these via digital communication. As these self-monitoring records were not submitted directly, we classified this BCT as delivered through a non-digital component. However, digital communication was used to Prompt/Cue completion of the self-testing behavior and monitor its completion in five studies (Katalenich et al., 2015; Leu et al., 2005; Magid et al., 2011; Nundy et al., 2013, 2014; Shane-McWhorter et al., 2014), although examining the impact on the self-testing behavior itself was not within the scope of this review.

The results from self-testing were used in five studies to deliver the BCT Monitoring outcome(s) of behavior by others without feedback, (Friedman et al., 1996; Moore et al., 2015; Piette et al., 2000, 2015; Shane-McWhorter et al., 2014) or Feedback on the outcomes of behavior in three studies (Bove et al., 2013; Magid et al., 2011; Vollmer et al., 2014). In the study by Katalenich et al. (2015) patient participants only received feedback if the results of the self-testing required action, otherwise they were monitored without feedback. This was also the case for the assessment of depressive symptoms in the studies by Aikens, Trivedi, Heapy, et al. (2015) and Kuusalo et al. (2020). In these cases, Monitoring of outcomes without feedback seemed to be for the benefit of clinicians as part of remote monitoring rather than being used to support reflective motivation of patients for taking medication. Only the study by Bender et al. (2010) delivered feedback on outcomes via automated two-way digital communication. 
Of the five studies which only monitored outcomes without feedback, two reported improvements in medication adherence (Friedman et al., 1996; Piette et al., 2000), although an additional study reported improvements in clinical outcomes (ShaneMcWhorter et al., 2014). All of these studies were for patient participants with diabetes. Of the three studies which only used Feedback on outcomes of behavior, (Bove et al., 2013; Magid et al., 2011; Vollmer et al., 2014) one found an improvement in medication adherence (Vollmer et al., 2014) and one in clinical outcomes (Magid et al., 2011). All three studies were for patient participants with cardiovascular disease. Of the five studies using a combination of both BCTs, three found an improvement in medication adherence, (Aikens, Rosland, et al., 2015; Aikens, Trivedi, Aron, et al., 2015; Bender et al., 2010; Katalenich et al., 2015) with the study by Katalenich et al. (2015) also finding an improvement in diabetes control, but neither result was statistically significant. All three studies coded as including the Self-monitoring of outcomes of bebavior BCT found improvements in medication adherence. However, it should be noted that only the study by Bender et al. (2010) explicitly seemed to link these health outcomes with taking medication; most of the interventions studied only described linking these outcomes to lifestyle influences, such as diet or exercise. Where Feedback on the outcomes of bebavior was delivered using a non-digital component, insufficient detail was provided to know what exactly was discussed.

Removing psychological and physical capability barriers to taking medication

Providing an automated two-way digital communication intervention has the potential to highlight psychological and physical capability barriers to taking medication. Including some form of 'live' interaction with a healthcare professional provides opportunities for patients to ask questions and healthcare professionals to assess the presence of these barriers. Where there was the opportunity for patient participants to have this assessment in a 'live' interaction, this was coded as the BCT Social support (unspecified) for the taking medication behavior. Seventeen studies included this BCT. However, details of what support was provided was often poorly described so coding further BCTs was not always possible. Five studies did describe providing some form of education to patient participants (Nundy et al., 2013, 2014; Piette et al., 2000; Shane-McWhorter et al., 2014; Sherrard et al., 2015; Spoelstra et al., 2016), which could have addressed psychological capability barriers and two referenced improving motivation (Bove et al., 2013; Nundy et al., 2013, 2014). However, most referenced identifying 'problems' or 'barriers' to 'selfmanagement' which could potentially include a wide range of medication taking issues.

\section{Asking for support to address psychological and physical capability gaps}

Six studies used the BCT Prompts/Cues to trigger a new behavior from patients which we categorised as asking for support (Aikens, Trivedi, Heapy, et al., 2015; Bender et al., 2010; Johnston et al., 2018; King, Kinvig, \& Steif, 2017; Magid et al., 2011; Nelson, Mulvaney, Gebretsadik, Ho, et al., 2016; Nelson, Mulvaney, Gebretsadik, Johnson, et al., 2016). Two studies also used digital communication to deliver the BCT Social support (practical) for asking for support by transferring the patient directly to a support line (Aikens, Trivedi, Heapy, et al., 2015) or linking this to a call-back request, (Magid et al., 2011) both using IVR technology. However, targeting the asking for support behavior had no clear effect on medication adherence with only three out of the seven studies targeting this behavior 
reporting an improvement in medication adherence (Bender et al., 2010; Cizmic et al., 2015; King et al., 2017) and one in clinical outcomes (Magid et al., 2011).

\section{Discussion}

Our findings demonstrate that automated two-way digital communication to improve medication adherence can target four different medication taking behaviors. While most interventions aimed to influence the behavior taking medication, the behavior obtaining medication had a greater association with improved medication adherence outcomes. Obtaining medication was targeted using a range of BCTs, and further research is needed to verify which BCTs are more or less effective.

The BCT most linked to results of increased medication adherence was Problem solving targeting taking medication. This BCT may facilitate the reduction of physical and/or psychological capability barriers to taking medication. Interventions which provided feedback on taking medication, even if this was conditional on identifying medication nonadherence, also seemed to be associated with improved medication adherence study results. This may also be due to the delivery of some form of problem solving, even if we could not code for this directly using the available descriptions. A recent meta-regression review has also found that patient participants 'reporting whether or not [taking medication] was performed' was positively associated with increases in intervention effect size for medication adherence in cardio-metabolic medicines (Kassavou \& Sutton, 2018), although they did not look at feedback on this separately.

We found that interventions providing feedback on health outcomes from taking medication could also be used to increase reflective motivation for medication taking, and this has been suggested in another review (Mistry et al., 2015). However, these outcomes were rarely linked directly to medicine taking, and therefore patients may not be encouraged to make a direct connection between taking medicines and health outcomes. Qualitative studies with patient participants have found that evaluating outcomes of medication taking may be an important motivator for medication adherence (Kassavou, Houghton, Edwards, Brimicombe, \& Sutton, 2019; Rathbone, Jamie, Todd, \& Husband, 2020). However, providing feedback on outcomes may be complicated by the impact of other variables, such as lifestyle choices.

We found that providing one-way messages delivering the BCT Information about bealth consequences did not seem to have a beneficial effect on medication adherence outcomes, although this is something which has been suggested as an effective strategy by others with cardio-metabolic medicines (Kassavou \& Sutton, 2017). Perceived necessity for medication has been found to be an important predictor of medication adherence (Horne \& Weinman, 1999), and is something which could be influenced using this BCT. Several validated self-report medication adherence tools incorporate a component of perceived necessity; however, as these are reported as composite scores, it was not possible to determine if changing medication perceptions was an outcome separate to overall adherence. Only one study measured this separately using the Beliefs about Medicines Questionnaire (BMQ) tool (Horne, Weinman, \& Hankins, 1999), and this study did find an improvement in positive medication beliefs as a result of their intervention (Bender et al., 2010). However, as this was used alongside a range of other BCTs, how best to persuade patients of the beneficial outcomes from taking medication is something that requires further investigation. 
Prompts/Cues aimed at taking medication was one of the most common BCTs included in digital communication interventions; however, we found that it did not seem to be connected to improving medication adherence outcomes. Other reviews evaluating digital communication reminders to improve medication adherence have found mixed results with some reporting a positive impact (Kannisto, Koivunen, \& Välimaki, 2014; Sarabi et al., 2016; Tran, Coffman, Sumino, \& Cabana, 2014) and others questioning their value (Free, Phillips, \& Galli, 2013; Jörntén-Karlsson, Pintat, Molloy-Bland, Berg, \& Ahlqvist, 2016; Mistry et al., 2015). Most of these reviews do not define what they consider to be a reminder, which could be one of several different BCTs from the taxonomy. One recent study allowed patient participants to choose whether they received reminders, (Nelson et al., 2020) and this might offer a better solution than routine incorporation of Prompts/Cues.

The model used to integrate automated two-way digital communication with nondigital intervention components varied among the included studies. Where there was the option of 'live' communication with healthcare professionals, we identified that SMS and IVR can be used to target the behavior asking for support for medication taking. We found no evidence that targeting the behavior asking for support had any impact on medication adherence or clinical outcomes in our review, but it was unclear what support was provided once patient participants sought additional help. In an article by Chiang, Guo, Amico, Atkins, \& Lester (2018) eight additional BCTs were identified based on the 'live' interaction which followed from patient participants responding to a two-way digital communication intervention. Other reviews have suggested that digital interventions should be supplemented with non-digital components (Ciciriello et al., 2013; Fenerty et al., 2012; Granger \& Bosworth, 2011; Hamine et al., 2015; Mistry et al., 2015). More explicit exploration of how non-digital intervention components contribute to overall effectiveness of interventions alongside automated two-way digital communication is an area for further research.

The behavior obtaining medication as a distinct behavior from taking medication has been identified in another review on digital communication to support medication adherence (Kassavou \& Sutton, 2018). However, our behavioral map extends the list of behaviors that interventions can target to include self-testing and asking for support with medicines taking. While other reviews have examined whether automated two-way digital communication is effective for supporting adherence to medicines (Hamine et al., 2015; Kassavou \& Sutton, 2018; Mistry et al., 2015), and others have included consideration of BCTs (Kassavou \& Sutton, 2018; Long, Bartlett, Farmer, \& French, 2019; Patton, Hughes, Cadogan, \& Ryan, 2017), we are the first to extend this to how the delivery of such BCTs can influence patients' capability, opportunity, and motivation to take their medication for long-term conditions.

Delivery of the BCTs which were found to be related to improvements in outcomes required two-way communication with the patient, and this is something that other reviewers have highlighted as important for digital communication intervention efficacy (Fjeldsoe et al., 2009; Granger \& Bosworth, 2011; Kassavou \& Sutton, 2018; Wald et al., 2015). A recent RCT examining one-way text messaging found no improvement in medication adherence for medicines used in secondary prevention of cardiovascular disease, further supporting this conclusion (Bermon, Uribe-Rodríguez, \& Pérez-Rivero, 2019). However, some BCTs are also more difficult to deliver in this clinical context, such as providing feedback on the outcomes of behavior. Guidance on designing text messaging programmes for health behavior change also suggests two-way messaging to 
promote engagement in interventions (Abroms, Whittaker, Free, Mendel Van Alstyne, \& Schindler-Ruwisch, 2015).

Other reviews have questioned the potential for automated digital communication to be successful at improving medication adherence (Mistry et al., 2015); however, our analysis suggests this is not necessarily a barrier. This review has helped identify potential behavioral components to be used in a future intervention using automated two-way text messaging to improve medication taking. Similar interventions are also in development, but have not chosen the same BCTs highlghted here as potentially helpful (Bartlett, Farmer, Rea, \& French, 2020; Kassavou et al., 2019). The findings of such studies will help shed further light on the validity of our conclusions.

We focused on older forms of technology (SMS and IVR). While some newer technologies, such as smart phone apps, may offer the opportunity to deliver a wider range of BCTs, any potential for these needs to be offset against the reduced accessibility of this technology, for example, requirement for an internet connection and more expensive handset.

\section{Limitations}

A limitation of our findings is that where behaviors are targeted in combination, the influence of each is not possible to separate. For example, some studies measure medication adherence using dispensing data, which are a measure of obtaining medication and not necessarily taking medication. A wide range of medication adherence measures were used within the included studies. Some of these were collected via the two-way digital communication intervention and as such have not been validated. Most measures of medication adherence also only provide an indirect measurement of medication consumption (Lam \& Fresco, 2015).

The lack of access to all intervention content also presents a previously identified challenge to the BCT coding process (Free, Phillips, \& Watson, 2013; Pfaeffli Dale, Dobson, Whittaker, \& Maddison, 2016). This was the case, in particular, when examining multiple studies using the same text messaging content, but where different example text messages were reported (Mayberry et al., 2017; Nelson, Mulvaney, Gebretsadik, Johnson, et al., 2016; Osborn \& Mulvaney, 2013). We used guidance in the the $\mathrm{BCW}$ to assess how the included BCTs seemed to influence medication taking behaviors as these descriptions were mostly lacking within the articles themsleves. More explicit descriptions about inclusion of BCTs and their intended mechanisms would help future synthesis, and an ontology of behavior change intervention mechanisms has recently been created which should facilitate this (Marques, Carey, \& Michie, 2021). Inability to compare findings across different studies has previously been highlighted as a limitation of research in the area of mobile health (Aranda-Jan, Mohutsiwa-Dibe, \& Loukanova, 2014). We have also not covered in this review the potential role of tailoring interventions which could also mediate potential effectiveness of BCTs and has been highlighted by others (Fjeldsoe et al., 2009; Nundy et al., 2014; Vervloet et al., 2012).

\section{Areas for future research}

More recently, the potential for mobile technologies to increase habit strength for medication taking has been highlighted (Badawy, Shah, Heneghan, \& Beg, 2020); however only three studies were coded in this review for including the Habit formation BCT. All three had positive outcomes, but further research is needed to see how 
automated two-way digital communication could further increase habit strength for taking medication, in particular, the frequency and length of contact required to affect this behavior in the longer term. Future research should also measure habit strength directly using tools, such as the Self-Reported Habit Index (Verplanken \& Orbell, 2013), to find out whether this is the mechanism by which providing feedback on behavior may improve medication adherence outcomes, and this is something which has also been highlighted by others (Badawy et al., 2020).

Only two studies included in this review targeted patient participants with more than one LTC. The growing prevalence of multimorbidity presents a major challenge for automated digital communication interventions, with or without non-digital intervention components. It is also unclear which healthcare professional group may be best placed to accompany digital communication interventions, and this will need to be explored alongside the role that they would play in increasing motivation or removing barriers to medication taking.

Future research should also examine the contexts which allow behavioral mechanisms for automated two-way digital communication interventions to work most effectively. This will add to our understanding about which patients should be offered these interventions, and in what circumstances. This would enable healthcare professionals to make informed decisions about what types of medication-related support patients need. This is important as one type of intervention will not be appropriate for everyone.

\section{Conclusion}

Automated two-way digital communication, such as SMS and IVR, have already been shown to have positive effects on medication adherence and be acceptable to patient participants. This article provides some explanation as to why two-way digital communication, with or without non-digital components, may be more effective than one-way communication. This was achieved by identifying the BCTs which can be delivered using this delivery format and the medication-related behaviors which can be targeted. This includes the use of digital communication to monitor performance of the taking medication behavior in order to detect barriers, such as those relating to physical or psychological capability. Using digital communication to support the monitoring of outcomes from the behavior taking medication may also work to increase reflective motivation for behavior performance. Removing physical opportunity as a barrier to taking medication by targeting the pre-requisite behavior, obtaining medication, may also be an important behavioral target, interventions where this is a barrier to medication adherence. Findings from this review can help inform the design of future automated twoway digital communication interventions by suggesting behavioral elements that may support improved medication adherence for patients self-managing their long-term conditions.

\section{Acknowledgements}

This study was funded by the National Institute for Health Research (NIHR) Doctoral Research Fellowship (2016-09-163). The views expressed are those of the author(s) and not necessarily those of the NIHR or the Department of Health and Social Care. 


\section{Conflict of interest}

All authors declare no conflict of interest.

\section{Author contribution}

Gemma Donovan: Conceptualization (equal); Formal analysis (equal); Funding acquisition (equal); Investigation (equal); Project administration (equal); Visualization (equal); Writing - original draft (equal); Writing - review \& editing (equal). Nicola Hall: Formal analysis (equal); Investigation (equal); Writing - review \& editing (equal). Jonathan Ling: Conceptualization (equal); Supervision (equal); Writing - review \& editing (equal). Felicity Smith: Conceptualization (equal); Supervision (equal); Writing review \& editing (equal). Scott Wilkes: Conceptualization (equal); Funding acquisition (equal); Supervision (equal); Writing - review \& editing (equal).

\section{Data availability statement}

Data sharing not applicable - no new data generated.

\section{References}

Abroms, L. C., Whittaker, R., Free, C., Mendel Van Alstyne, J., \& Schindler-Ruwisch, J. M. (2015). Developing and pretesting a text messaging program for health behavior change: Recommended steps. JMIR mHealth and uHealth, 3, e107. https://doi.org/10.2196/mhealth.4917

Aikens, J. E., Rosland, A.-M., \& Piette, J. D. (2015). Improvements in illness self-management and psychological distress associated with telemonitoring support for adults with diabetes. Prim Care Diabetes, 9, 127-134. https://doi.org/10.1016/j.pcd.2014.06.003

Aikens, J. E., Trivedi, R., Aron, D. C., \& Piette, J. D. (2015). Integrating support persons into diabetes telemonitoring to improve self-management and medication adherence. Journal of General Internal Medicine, 30, 319-326. https://doi.org/10.1007/s11606-014-3101-9

Aikens, J. E., Trivedi, R., Heapy, A., Pfeiffer, P. N., \& Piette, J. D. (2015). Potential impact of incorporating a patient-selected support person into mHealth for depression. Journal of General Internal Medicine, 30, 797-803. https://doi.org/10.1007/s11606-015-3208-7

Anglada-Martinez, H., Riu-Viladoms, G., Martin-Conde, M., Rovira-Illamola, M., Sotoca-Momblona, J. M., \& Codina-Jane, C. (2015). Does mHealth increase adherence to medication? Results of a systematic review. International Journal of Clinical Practice, 69(1), 9-32. https://doi.org/10. $1111 /$ ijcp. 12582

Aranda-Jan, C. B., Mohutsiwa-Dibe, N., \& Loukanova, S. (2014). Systematic review on what works, what does not work and why of implementation of mobile health (mHealth) projects in Africa. BMC Public Health, 14(1), 188. https://doi.org/10.1186/1471-2458-14-188

Badawy, S. M., Shah, R., Beg, U., \& Heneghan, M. B. (2020). Habit strength, medication adherence, and habit-based mobile health interventions across chronic medical conditions: Systematic review. Journal of Medical Internet Research, 22(4), e17883. https://doi.org/10.2196/17883

Kiera, B. Y., Andrew, F., Rustam, R., \& French David, P. (2020). Use of brief messages based on behavior change techniques to encourage medication adherence in people with type 2 diabetes: Developmental studies. Journal of Medical Internet Research, 22(5), e15989. https://doi.org/ $10.2196 / 15989$

Bender, B. G., Apter, A., Bogen, D. K., Dickinson, P., Fisher, L., Wamboldt, F. S., \& Westfall, J. M. (2010). Test of an interactive voice response intervention to improve adherence to controller medications in adults with asthma. The Journal of the American Board of Family Medicine, 23, 159-165. https://doi.org/10.3122/jabfm.2010.02.090112 
Bermon, A., Uribe, A. F., Pérez-Rivero, P. F., Prieto-Merino, D., Saaibi, J. F., Silva, F. A., . . Perel, P. (2021). Efficacy and safety of text messages targeting adherence to cardiovascular medications in secondary prevention: TXT2HEART Colombia randomized controlled trial. JMIR mHealth and uHealth, 9(7), e25548. https://doi.org/10.2196/25548

Boker, A., Feetham, H. J., Armstrong, A., Purcell, P., \& Jacobe, H. (2012). Do automated text messages increase adherence to acne therapy? Results of a randomized, controlled trial.Journal of the American Academy of Dermatology, 67, 1136-1142. https://doi.org/10.1016/j.jaad. 2012.02.031

Boland, M. V., Chang, D. S., Frazier, T., Plyler, R., Jefferys, J. L., \& Friedman, D. S. (2014). Electronic monitoring to assess adherence with once-daily glaucoma medications and risk factors for nonadherence: The automated dosing reminder study. JAMA Ophthalmology, 132, 845-850. https://doi.org/10.1001/jamaophthalmol.2014.857

Bove, A. A., Homko, C. J., Santamore, W. P., Kashem, M., Kerper, M., \& Elliott, D. J. (2013). Managing hypertension in urban underserved subjects using telemedicine-A clinical trial. American Heart Journal, 165, 615-621. https://doi.org/10.1016/j.ahj.2013.01.004

Chiang, N., Guo, M., Amico, K. R., Atkins, L., \& Lester, R. T. (2018). Interactive two-way mHealth interventions for improving medication adherence: An evaluation using the behaviour change wheel framework. JMIR mHealth and uHealth, 6, e87. https://doi.org/10.2196/mhealth.9187

Ciciriello, S., Johnston, R. V., Osborne, R. H., Wicks, I., deKroo, T., Clerehan, R., . . Buchbinder, R. (2013). Multimedia educational interventions for consumers about prescribed and over-thecounter medications. Cochrane Database of Systematic Reviews, 4, CD008416. https://doi. org/10.1002/14651858.cd008416.pub2

Cizmic, A. D., Heilmann, R. M. F., Milchak, J. L., Riggs, C. S., \& Billups, S. J. (2015). Impact of interactive voice response technology on primary adherence to bisphosphonate therapy: a randomized controlled trial. Osteoporosis International, 26, 2131-2136. https://doi.org/10. 1007/s00198-015-3116-z

Cutler, R. L., Fernandez-Llimos, F., Frommer, M., Benrimoj, C., \& Garcia-Cardenas, V. (2018). Economic impact of medication non-adherence by disease groups: A systematic review. British Medical Journal Open, 8(1), 1-13. https://doi.org/10.1136/bmjopen-2017-016982

DeKoekkoek, T., Given, B., Given, C. W., Ridenour, K., Schueller, M., \& Spoelstra, S. L. (2015). mHealth SMS text messaging interventions and to promote medication adherence: An integrative review. Journal of Clinical Nursing, 24, 2722-2735. https://doi.org/10.1111/ jocn. 12918

Easthall, C., \& Barnett, N. (2017). Using theory to explore the determinants of medication adherence; Moving away from a one-size-fits-all approach. Pharmacy, 5, 50. https://doi.org/10. 3390/pharmacy5030050

Fang, K. Y., Maeder, A. J., \& Bjering, H. (2016). Current trends in electronic medication reminders for self care. Studies in Health Technology and Informatics, 231, 31-41.

Fat, L. N. (2018). Health survey for England 2017 - adult bealth (co-morbidities). Retrieved from https://files.digital.nhs.uk/8E/8907A5/HSE17-Adult-Health-rep.pdf. Accessed September 11, 2020.

Fenerty, S. D., West, C., Davis, S. A., Kaplan, S. G., \& Feldman, S. R. (2012). The effect of reminder systems on patients' adherence to treatment. Patient Prefer Adberence, 6, 127-135. https://doi. org/10.2147/PPA.S26314

Fjeldsoe, B. S., Marshall, A. L., \& Miller, Y. D. (2009). Behavior change interventions delivered by mobile telephone short-message service. American Journal of Preventive Medicine, 36, 165173. https://doi.org/10.1016/j.amepre.2008.09.040

Free, C., Phillips, G., Galli, L., Watson, L., Felix, L., Edwards, P., ... Haines, A. (2013). The effectiveness of mobile-health technology-based health behaviour change or disease management interventions for health care consumers: A systematic review. PLoS Med, 1O(1), e1001362. https://doi.org/10.1371/journal.pmed.1001362

Free, C., Phillips, G., Watson, L., Galli, L., Felix, L., Edwards, P., ... Haines, A. (2013). The effectiveness of mobile-health technologies to improve health care service delivery processes: A 
systematic review and meta-analysis. PLoS Med, 10(1), e1001363. https://doi.org/10.1371/ journal.pmed.1001363

Friedman, R. (1996). A telecommunications system for monitoring and counseling patients with hypertension impact on medication adherence and blood pressure control. American Journal of Hypertension, 9(4), 285-292. https://doi.org/10.1016/0895-7061(95)00353-3

Garofalo, R., Kuhns, L., Hotton, A., Johnson, A., Muldoon, A., \& Rice, D. (2016). A randomized controlled trial of personalized text message reminders to promote medication adherence among HIV-positive adolescents and young adults. AIDS and Behavior, 20, 1049-1059. https:// doi.org/10.1007/s10461-015-1192-x

Glanz, K., Beck, A. D., Bundy, L., Primo, S., Lynn, M. J., Cleveland, J., .. Echt, K. V. (2012). Impact of a health communication intervention to improve glaucoma treatment adherence: Results of the interactive study to increase glaucoma adherence to treatment trial. Archives of Ophthalmology, 130, 1252-1258. https://doi.org/10.1001/archophthalmol.2012.1607

Google LLC (2018) Google forms. Retrieved from https://www.google.co.uk/forms/about/

Granger, B. B., \& Bosworth, H. (2011). Medication adherence: Emerging use of technology. Current Opinion in Cardiology, 26, 279-287. https://doi.org/10.1002/ana.22528.Toll-like

Hamine, S., Gerth-Guyette, E., Faulx, D., Green, B. B., \& Ginsburg, A. S. (2015). Impact of mHealth chronic disease management on treatment adherence and patient outcomes: A systematic review. Journal of Medical Internet Research, 17(2), 1-15. https://doi.org/10.2196/jmir.3951

Harris, L. T., Lehavot, K., Huh, D., Yard, S., Andrasik, M. P., Dunbar, P. J., \& Simoni, J. M. (2010). Twoway text messaging for health behavior change among human immunodeficiency virus-positive individuals. Telemedicine and e-Health, 16, 1024-1029. https://doi.org/10.1089/tmj.2010. 0050

Horne, R., \& Weinman, J. (1999). Patients' beliefs about prescribed medicines and their role in adherence to treatment in chronic physical illness. Journal of Psychosomatic Research, 47, 555-567. https://doi.org/10.1016/S0022-3999(99)00057-4

Horne, R., Weinman, J., \& Hankins, M. (1999). The beliefs about medicines questionnaire: The development and evaluation of a new method for assessing the cognitive representation of medication. Psychology Health, 14(1), 1-24. https://doi.org/10.1080/08870449908407311

Iribarren, S. J., Brown, 3rd, W., Giguere, R., Stone, P., Schnall, R., Staggers, N., \& CarballoDiéguez, A. (2017). Scoping review and evaluation of SMS/text messaging platforms for mHealth projects or clinical interventions. International Journal of Medical Informatics, 101, 28-40. https://doi.org/10.1016/j.ijmedinf.2017.01.017

Jackson, C., Eliasson, L., Barber, N., \& Weinman, J. (2014). Applying COM-B to medication adherence. The European Health Psychologist, 16(1), 7-17.

Johnston, J. C., van der Kop Mia, L., Kirsten, S., Gina, O., Fawziah, M., Mohsen, S., . . Lester, R. T. (2018). The effect of text messaging on latent tuberculosis treatment adherence: a randomised controlled trial. European Respiratory Journal, 51(2), 1701488. https://doi.org/10.1183/ 13993003.01488-2017

Jörntén-Karlsson, M., Pintat, S., Molloy-Bland, M., Berg, S., \& Ahlqvist, M. (2016). Patient-centered interventions to improve adherence to statins: A narrative synthesis of systematically identified studies. Drugs, 76(15), 1447-1465. https://doi.org/10.1007/s40265-016-0640-x

Kannisto, K. A., Koivunen, M. H., \& Välimaki, M. A. (2014). Use of mobile phone text message reminders in health care services: A narrative literature review. Journal of Medical Internet Research, 16, e222. https://doi.org/10.2196/jmir.3442

Kassavou, A., Houghton, V., Edwards, S., Brimicombe, J., \& Sutton, S. (2019). Development and piloting of a highly tailored digital intervention to support adherence to antihypertensive medications as an adjunct to primary care consultations. British Medical Journal Open, 9(1), e024121. https://doi.org/10.1136/bmjopen-2018-024121

Kassavou, A., \& Sutton, S. (2017). Reasons for non-adherence to cardiometabolic medications, and acceptability of an interactive voice response intervention in patients with hypertension and type 2 diabetes in primary care: A qualitative study. British Medical Journal Open, 7(8), e015597. https://doi.org/10.1136/bmjopen-2016-015597 
Kassavou, A., \& Sutton, S. (2018). Automated telecommunication interventions to promote adherence to cardio-metabolic medications: Meta-analysis of effectiveness and meta-regression of behaviour change techniques. Health Psychology Review, 12(1), 25-42. https://doi.org/10. 1080/17437199.2017.1365617

Katalenich, B., Shi, L., Liu, S., Shao, H., McDuffie, R., Carpio, G., . . Fonseca, V. (2015). Evaluation of a remote monitoring system for diabetes control. Clinical Therapeutics, 37, 1216-1225. https:// doi.org/10.1016/j.clinthera.2015.03.022

King, E., Kinvig, K., Steif, J., Qiu, A. Q., Maan, E. J., Albert, A. Y., .. Murray, M. (2017). Mobile text messaging to improve medication adherence and viral load in a vulnerable Canadian population living with human immunodeficiency virus: A repeated measures study. Journal of Medical Internet Research, 19, e190. https://doi.org/10.2196/jmir.6631

Kuusalo, L., Sokka-Isler, T., Kautiainen, H., Ekman, P., Kauppi, M. J., Pirilä, L., . . Puolakka, K. (2020). Automated text message-enhanced monitoring versus routine monitoring in early rheumatoid arthritis: A randomized trial. Arthritis Care \& Research, 72(3), 319-325. https://doi.org/10. 1002/acr.23846

Lam, W. Y., \& Fresco, P. (2015). Medication adherence measures: An overview. BioMed Research International, 2015, 1-12. https://doi.org/10.1155/2015/217047

Lee, H., Ralston, D., Beautrais, A. L., \& Larkin, G. L. (2014). Text messaging interventions for promoting medication adherence: A review of the literature. International Journal of General Medicine and Pharmacy, 3(2), 79-100.

Leu, M. G., Norris, T. E., Hummel, J., Isaac, M., \& Brogan, M. W. (2005). A randomized, controlled trial of an automated wireless messaging system for diabetes. Diabetes Technology \& Therapeutics, 7, 710-718. https://doi.org/10.1089/dia.2005.7.710

Linn, A. J., Vervloet, M., van Dijk, L., Smit, E. G., \& Van Weert, J. C. M. (2011). Effects of eHealth interventions on medication adherence: A systematic review of the literature. Journal of Medical Internet Research, 13, e103. https://doi.org/10.2196/jmir.1738

Long, H., Bartlett, Y. K., Farmer, A. J., \& French, D. P. (2019). Identifying brief message content for interventions delivered via mobile devices to improve medication adherence in people with type 2 diabetes mellitus: A rapid systematic review. Journal of Medical Internet Research, 21 (1), e10421. https://doi.org/10.2196/10421

Magid, D. J., Ho, P. M., Olson, K. L., Brand, D. W., Welch, L. K., Snow, K. E., , . Havranek, E. P. (2011). A multimodal blood pressure control intervention in 3 healthcare systems. American Journal of Managed Care, 17, e96-e103.

Michie, S., Johnston, M., Rothman, A. J., de Bruin, M., Kelly, M. P., Carey, R. N., .. Zink, S. (2021). Developing an evidence-based online method of linking behaviour change techniques and theoretical mechanisms of action: A multiple methods study. Health Services and Delivery Research, 9(1), 1-168. https://doi.org/10.3310/hsdr09010

Mayberry, L. S. S., Mulvaney, S. A. A., \& Johnson, K. B. B. (2017). The MEssaging for diabetes intervention reduced barriers to medication adherence among low-income, diverse adults with type 2. Journal of Diabetes Science and Technology, 11(1), 92-99. http://dst.sagepub.com/ content/by/year

Michie, S., Atkins, L., \& West, R. (2014). Understand the behaviour. The behaviour change wheel a guide to designing interventions (pp. 31-106). Surrey: Silverback Publishing.

Michie, S., Richardson, M., Johnston, M., Abraham, C., Francis, J., Hardeman, W., ... Wood, C. E. (2013). The behavior change technique taxonomy (v1) of 93 hierarchically clustered techniques: Building an international consensus for the reporting of behavior change interventions. Annals of Behavioral Medicine, 46(1), 81-95. https://doi.org/10.1007/ s12160-013-9486-6

Michie, S., van Stralen, M. M., \& West, R. (2011). The behaviour change wheel: A new method for characterising and designing behaviour change interventions. Implementation Science, 6, 42. https://doi.org/10.1186/1748-5908-6-42

Mistry, N., Keepanasseril, A., Wilczynski, N. L., Nieuwlaat, R., Ravall, M., Haynes, R. B. \& Patient Adherence Review Team (2015). Technology-mediated interventions for enhancing medication 
adherence. Journal of the American Medical Informatics Association, 22(e1), e177-e193. https://doi.org/10.1093/jamia/ocu047

Moody, A., Mindell, J., \& Faulding, S. (2016). Health survey for England 2016 prescribed medicines. Leeds, UK: NHS Digital. https://files.digital.nhs.uk/pdf/3/c/hse2016-pres-med.pdf

Moore, D. J., Poquette, A., Casaletto, K. B., Gouaux, B., Montoya, J. L., \& Posada, C. ... HIV Neurobehavioral Research Program (HNRP) Group (2015). Individualized texting for adherence building (iTAB): improving antiretroviral dose timing among HIV-infected persons with cooccurring bipolar disorder. AIDS and Behavior, 19, 459-471. https://doi.org/10.1007/s10461014-0971-0

Morisky, D. E., Levine, D. M., Green, L. W., Shapiro, S., Russell, R. P., \& Smith, C. R. (1983). Five-year blood pressure control and mortality following health education for hypertensive patients. American Journal of Public Health, 73(2), 153-162. https://doi.org/10.2105/AJPH.73.2.153

Nelson, L. A., Mulvaney, S. A., Gebretsadik, T., Ho, Y. X., Johnson, K. B., \& Osborn, C. Y. (2016). Disparities in the use of a mHealth medication adherence promotion intervention for lowincome adults with type 2 diabetes. Journal of the American Medical Informatics Association, 23(1), 12-18. https://doi.org/10.1093/jamia/ocv082

Nelson, L., Mulvaney, S., Gebretsadik, T., Johnson, K., \& Osborn, C. (2016). The MEssaging for Diabetes (MED) intervention improves short-term medication adherence among low-income adults with type 2 diabetes. Journal of Behavioral Medicine, 39, 995-1000. https://doi.org/10. 1007/s10865-016-9774-2

Nelson Lyndsay, A., Andrew, S., Robert, G., LeStourgeon, L. M., Wallston Kenneth, A., \& Mayberry Lindsay, S. (2020). User engagement among diverse adults in a 12-month text message-delivered diabetes support intervention: Results from a randomized controlled trial. JMIR mHealth and uHealth, 8(7), e17534. https://doi.org/10.2196/17534

Nieuwlaat, R., Wilczynski, N., Navarro, T., Hobson, N., Jeffery, R., Keepanasseril, A., . . Haynes, R. B. (2014). Interventions for enhancing medication adherence. Cochrane Database of Systematic Reviews, 2014, CD000011. https://doi.org/10.1002/14651858.cd000011.pub4

Nundy, S., Dick, J. J., Solomon, M. C., \& Peek, M. E. (2013). Developing a behavioral model for mobile phone-based diabetes interventions. Patient Education and Counseling, 90(1), 125132. https://doi.org/10.1016/j.pec.2012.09.008

Nundy, S., Mishra, A., Hogan, P., Lee, S. M., Solomon, M. C., \& Peek, M. E. (2014). How do mobile phone diabetes programs drive behavior change? Evidence from a mixed methods observational cohort study. The Diabetes Educator, 40, 806-819. https://doi.org/10.1177/ 0145721714551992

Osborn, C. Y., \& Mulvaney, S. A. (2013). Development and feasibility of a text messaging and interactive voice response intervention for low-income, diverse adults with type 2 diabetes mellitus. Journal of Diabetes Science and Technology, 7, 612-622. https://doi.org/10.1177/ 193229681300700305

Page, M. J., McKenzie, J. E., Bossuyt, P. M., Boutron, I., Hoffmann, T. C., Mulrow, C. D., , . Moher, D. (2020). The PRISMA 2020 statement: An updated guideline for reporting systematic reviews. BMJ, 2021, 372. https://doi.org/10.1136/bmj.n71

Park, L. G., Howie-Esquivel, J., Chung, M. L., \& Dracup, K. (2014). A text messaging intervention to promote medication adherence for patients with coronary heart disease: A randomized controlled trial. Patient Education and Counseling, 94, 261-268. https://doi.org/10.1016/j. pec.2013.10.027

Park, L. G., Howie-Esquivel, J., \& Dracup, K. (2014). A quantitative systematic review of the efficacy of mobile phone interventions to improve medication adherence. Journal of Advanced Nursing, 68, 1932-1953. https://doi.org/10.1111/jan.12400

Park, L. G., Howie-Esquivel, J., \& Dracup, K. (2014). A quantitative systematic review of the efficacy of mobile phone interventions to improve medication adherence. Journal of Advanced Nursing, 70, 1932-1953. https://doi.org/10.1111/jan.12400

Park, L. G., Howie-Esquivel, J., Whooley, M. A., \& Dracup, K. (2015). Psychosocial factors and medication adherence among patients with coronary heart disease: A text messaging 
intervention. European Journal of Cardiovascular Nursing, 14, 264-273. https://doi.org/10. $1177 / 1474515114537024$

Patton, D. E., Hughes, C. M., Cadogan, C. A., \& Ryan, C. A. (2017). Theory-based interventions to improve medication adherence in older adults prescribed polypharmacy: A systematic review. Drugs and Aging, 34(2), 97-113. https://doi.org/10.1007/s40266-016-0426-6

Pfaeffli Dale, L., Dobson, R., Whittaker, R., \& Maddison, R. (2016). The effectiveness of mobilehealth behaviour change interventions for cardiovascular disease self-management: A systematic review. European Journal of Preventive Cardiology, 23, 801-817. https://doi.org/10.1177/ 2047487315613462

Pfaeffli Dale, L., Whittaker, R., Jiang, Y., Stewart, R., Rolleston, A., \& Maddison, R. (2015). Text message and internet support for coronary heart disease self-management: Results from the text 4 heart randomized controlled trial.Journal of Medical Internet Research, 17, e237. https:// doi.org/10.2196/jmir.4944

Piette, J. D., Striplin, D., Marinec, N., Chen, J., Trivedi, R. B., Aron, D. C., ... Aikens, J. E. (2015). A Mobile health intervention supporting heart failure patients and their informal caregivers: A randomized comparative effectiveness trial. Journal of Medical Internet Research, 17, e142. https://doi.org/10.2196/jmir.4550

Piette, J. D., Weinberger, M., McPhee, S. J., Mah, C. A., \& Kraemer, F. B. (2000). Do automated calls with nurse follow-up improve self-care and glycemic control among vulnerable patients with diabetes? American Journal of Medicine, 108(1), 20-27. https://doi.org/10.1016/S0002-9343 (99)00298-3

Pluye, P., Gagnon, M. P., Griffiths, F., \& Johnson-Lafleur, J. (2009). A scoring system for appraising mixed methods research, and concomitantly appraising qualitative, quantitative and mixed methods primary studies in Mixed Studies Reviews. International Journal of Nursing Studies, 46, 529-546. https://doi.org/10.1016/j.ijnurstu.2009.01.009

QSR International Pty Ltd. NVivo (2018). Retrieved from https:/www.qsrinternational.com/nvivoqualitative-data-analysis-software/home

Rathbone, A. P., Jamie, K., Todd, A., \& Husband, A. (2020). A qualitative study exploring the lived experience of medication use in different disease states: Linking experiences of disease symptoms to medication adherence. Journal of Clinical Pharmacy and Therapeutics, 46, 352362. https://doi.org/10.1111/jcpt.13288

Sarabi, R. E., Sadoughi, F., Orak, R. J., \& Bahaadinbeigy, K. (2016). The effectiveness of mobile phone text messaging in improving medication adherence for patients with chronic diseases: A systematic review. Iranian Red Crescent Medical Journal, 18, e25183. https://doi.org/10. 5812/ircmj. 25183

Sarkar, S., \& Sivashankar, P. (2015). Mobile SMS reminders for increasing medication adherence. International Journal of Pharmaceutical Sciences Review and Research, 32(1), 228-237.

Shane-McWhorter, L., Lenert, L., Petersen, M., Woolsey, S., McAdam-Marx, C., Coursey, J. M., ... Chuy, L. (2014). The utah remote monitoring project: Improving health care one patient at a time. Diabetes Technology \& Therapeutics, 16, 653-660. https://doi.org/10.1089/dia.2014. 0045

Sherrard, H., Duchesne, L., Wells, G., Kearns, S. A., \& Struthers, C. (2015). Using interactive voice response to improve disease management and compliance with acute coronary syndrome best practice guidelines: A randomized controlled trial. Canadian Journal of Cardiovascular Nursing, 25(1), 10-15.

Sherrard, H., Struthers, C., Kearns, S., Wells, G., \& Mesana, T. (2009). Using technology to create a medication safety net for cardiac surgery patients: A nurse-led randomized control trial. Canadian Journal of Cardiovascular Nursing, 19, 9-15.

Simoni, J. M., Huh, D., Frick, P. A., Pearson, C. R., Andrasik, M. P., Dunbar, P. J., \& Hooton, T. M. (2009). Peer support and pager messaging to promote antiretroviral modifying therapy in Seattle: A randomized controlled trial. Journal of Acquired Immune Deficiency Syndromes, 52, 465-473. https://doi.org/10.1097/QAI.0b013e3181b9300c 
Simple Shared Healthcare Ltd (2020). Simple telehealth. Retrieved from http://www.simple.uk.net/ . Accessed May 13, 2020

Spoelstra, S. L., Given, C. W., Sikorskii, A., Coursaris, C. K., Majumder, A., DeKoekkoek, T., .. Given, B. A. (2016). Proof of concept of a mobile health short message service text message intervention that promotes adherence to oral anticancer agent medications: a randomized controlled trial. Telemedicine and e-Health, 22, 497-506. https://doi.org/10.1089/tmj.2015.0126

Stacy, J. N., Schwartz, S. M., Ershoff, D., \& Shreve, M. S. (2009). Incorporating tailored interactive patient solutions using interactive voice response technology to improve statin adherence: results of a randomized clinical trial in a managed care setting. Population Health Management, 12, 241-254. https://doi.org/10.1089/pop.2008.0046

Stuart, G. W., Laraia, M. T., Ornstein, S. M., \& Nietert, P. J. (2003). An interactive voice response system to enhance antidepressant medication compliance. Topics in Health Information Management, 24(1), 15-20.

Tao, D., Xie, L., Wang, T., \& Wang, T. (2015). A meta-analysis of the use of electronic reminders for patient adherence to medication in chronic disease care. Journal of Telemedicine and Telecare, 21(1), 3-13. https://doi.org/10.1177/1357633X14541041

Thakkar, J., Kurup, R., Laba, T. L., Santo, K., Thiagalingam, A., Rodgers, A., . . Chow, C. K. (2016). Mobile telephone text messaging for medication adherence in chronic disease a meta-analysis. JAMA Internal Medicine, 176, 340-349. https://doi.org/10.1001/jamainternmed.2015.7667

The World Bank (2020). Historical classification by income in XLS format. Retrieved from http:// databank.worldbank.org/data/download/site-content/OGHIST.xls. Accessed November 9, 2021.

Tran, N., Coffman, J. M., Sumino, K., \& Cabana, M. D. (2014). Patient reminder systems and asthma medication adherence: a systematic review. Journal of Asthma, 51, 536-543. https://doi.org/ $10.3109 / 02770903.2014 .888572$

Trautner, C., Richter, B., \& Berger, M. (1993). Cost-effectiveness of a structured treatment and teaching programme on asthma. European Respiratory Journal, 6, 1485-1491.

Tucker, J. A., Simpson, C. A., Huang, J., Roth, D. L., \& Stewart, K. E. (2013). Utility of an interactive voice response system to assess antiretroviral pharmacotherapy adherence among substance users living with HIV/AIDS in the rural South. AIDS Patient Care STDS, 27, 280286. https://doi.org/10.1089/apc.2012.0322

Verplanken, B., \& Orbell, S. (2003). Reflections on past behavior: A self-report index of zabit strength. Journal of Applied Social Psychology, 33(6), 1313-1330. https://doi.org/10.1111/j. 1559-1816.2003.tb01951.x

Vervloet, M., Linn, A. J., van Weert, J. C., de Bakker, D. H., Bouvy, M. L., \& van Dijk, L. (2012). The effectiveness of interventions using electronic reminders to improve adherence to chronic medication: a systematic review of the literature. Journal of the American Medical Informatics Association, 19, 696-704. https://doi.org/10.1136/amiajnl-2011-000748

Vollmer, W. M., Feldstein, A., Smith, D. H., Dubanoski, J. P., Waterbury, A., Schneider, J. L., . . Rand, C. (2011). Use of health information technology to improve medication adherence. American Journal of Managed Care, 17(Spec. Issue), SP79-SP87.

Vollmer, W. M., Owen-Smith, A. A., Tom, J. O., Laws, R., Ditmer, D. G., Smith, D. H., .. Rand, C. S. (2014). Improving adherence to cardiovascular disease medications with information technology. American Journal of Managed Care, 20(Spec No. 17), SP502-SP510.

Wald, D. S., Bestwick, J. P., Raiman, L., Brendell, R., \& Wald, N. J. (2014). Randomised trial of text messaging on adherence to cardiovascular preventive treatment (INTERACT trial). PLoS One, 9, e114268. https://doi.org/10.1371/journal.pone.0114268

Wald, D. S., Butt, S., Bestwick, J. P. \& Bestwick, J. P. (2015). One-way versus two-way text messaging on improving medication adherence: meta-analysis of randomized trials. The American Journal of Medicine, 128, 1139.e1-1139.e5. https://doi.org/10.1016/j.amjmed. 2015.05.035

World Health Organization (2003). Adberence to long-term therapies evidence for action. Geneva, Switzerland: World Health Organisation. 
Yard, S. S., Huh, D., King, K. M., \& Simoni, J. M. (2011). Patient-level moderators of the efficacy of peer support and pager reminder interventions to promote antiretroviral adherence. AIDS and Behavior, 15, 1596-1604. https://doi.org/10.1007/s10461-011-0001-4

Zabinski, R. A., Skinner, E. P., \& Buysman, E. K. (2012). Addressing gaps in care: Impact of barrierspecific medication adherence intervention. Am J Pharm Benefits, 4, e109-e117.

Received 23 July 2021; revised version received 28 November 2021

\section{Supporting Information}

The following supporting information may be found in the online edition of the article:

Appendix S1. Search strategy, inclusion and exclusion crtieria.

Appendix S2. TIMELY data extraction form for narrative synthesis systematic review.

Appendix S3. TIMELY narrative synthesis behavior change coding manual.

Appendix S4. Quality appraisal of included studies using the Mixed Methods Appraisal Tool (MMAT) v1.

Appendix S5. Summary results table for the paper: Influencing medication taking behaviours using automated two-way digital communication: A narrative synthesis. 